\title{
Configural processing in autism and its relationship to face processing
}

\author{
Marlene Behrmann ${ }^{\mathrm{a}, *}$, Galia Avidan ${ }^{\mathrm{a}}$, Grace Lee Leonard ${ }^{\mathrm{a}}$, Rutie Kimchi ${ }^{\mathrm{b}}$, \\ Beatriz Luna ${ }^{\mathrm{c}}$, Kate Humphreys ${ }^{\mathrm{a}}$, Nancy Minshew ${ }^{\mathrm{d}}$ \\ a Department of Psychology, Carnegie Mellon University, Pittsburgh, PA 15213-3890, USA \\ ${ }^{\mathrm{b}}$ Department of Psychology, University of Haifa, Haifa, Israel \\ ${ }^{\mathrm{c}}$ Departments of Psychiatry and Psychology, University of Pittsburgh, Pittsburgh, PA, USA \\ ${ }^{\mathrm{d}}$ Departments of Psychiatry and Neurology, University of Pittsburgh School of Medicine, Pittsburgh, PA, USA
}

Received 29 April 2004; received in revised form 31 March 2005; accepted 8 April 2005

Available online 23 May 2005

\begin{abstract}
Studies of the perceptual performance of individuals with autism have focused, to a large extent, on two domains of visual behavior, one associated with face processing and the other associated with global or holistic processing. Whether autistic individuals differ from neurotypical individuals in these domains is debatable and, moreover, the relationship between the behaviors in these two domains remains unclear. We first compared the face processing ability of 14 adult individuals with autism with that of neurotypical controls and showed that the autistic individuals were slowed in their speed of face discrimination. We then showed that the two groups differed in their ability to derive the global whole in two different tasks, one using hierarchical compound letters and the other using a microgenetic primed matching task with geometric shapes, with the autistic group showing a bias in favor of local information. A significant correlation was also observed between performance on the face task and the configural tasks. We then confirmed the prediction that the ability to derive the global whole is not only critical for faces but also for other objects as well, as the autistic individuals performed more slowly than the control group in discriminating between objects. Taken together, the results suggest that the bias for local processing seen in autistic individuals might have an adverse impact on their ability to process faces and objects.
\end{abstract}

(C) 2005 Elsevier Ltd. All rights reserved.

Keywords: Local; Global; Visual processing; Objects; Greebles; Faces; Configural

\section{Introduction}

"And this is how I recognize someone if I don't know who they are. I see what they are wearing, or if they have a walking stick, or funny hair, or a certain type of glasses, or they have a particular way of moving their arms, and I do a Search through my memories to see if I have met them before." (Christopher, the 15-year old autistic protagonist in The Curious Incident of the Dog in the Night-time by Mark Haddon).

"I often get into embarrassing situations because I do not remember faces unless I have seen the people many times or they have a very distinct facial feature such as a big beard,

\footnotetext{
* Corresponding author. Tel.: +1 412268 2790; fax: +1 4122682798.

E-mail address: behrmann@cnbc.cmu.edu (M. Behrmann).
}

thick glasses or a strange hairstyle.”(in “Thinking in Pictures and Other Reports from my Life with Autism" by Temple Grandin).

Autism is a developmental disorder that is associated with a number of characteristic deficits, most notably in the domains of social interaction, communication and imaginative behavior (Frith, 2003; Klin, Jones, Schultz, Volkmar, \& Cohen, 2002; Volkmar, Lord, Bailey, Schultz, \& Klin, 2004). It is further defined by the finding that most autistic individuals exhibit a restricted and repetitive behavioral repertoire. Abnormalities in visual processing have also been documented in autism (Kanner, 1943; Society for Autistic Children, 1978), although the nature and extent of the visuoperceptual impairment in these individuals remains a topic of debate. Many previous studies have focused on two visuoperceptual behaviors 
in autism, one related to face processing and the other related to the derivation of organized wholes from perceptual parts. While both lines of investigation have been fruitful, each set of findings remains somewhat controversial and, moreover, there has only been minimal consideration of the relationship between these behaviors in autism. We consider some of the existing data from each domain and then the possible relationship between them before we outline the current studies.

Many studies of face processing in children with autism have demonstrated the presence of an impairment that is widespread and present from an early age (Dawson et al., 2002), affecting both the perception of and the memory for faces (Ellis, Ellis, Fraser, \& Deb, 1994; Hauck, Fein, Maltby, Waterhouse, \& Feinstein, 1998; Klin et al., 1999; Langdell, 1977). The perceptual difficulties also affect the perception of the affect of faces (Hobson, 1986; Hobson, Ouston, \& Lee, 1988), the perception of direction of gaze (Jolliffe \& BaronCohen, 1997) and sometimes even the perception of gender (Hobson, 1987; Njiokiktjien et al., 2001). The same is true for adults with autism, although the impairment is apparently less severe in older individuals and in more cognitively able individuals (Boucher \& Lewis, 1992). The reported decrement in face processing is consistent with a series of recent functional imaging studies demonstrating atypical or weak activation of the fusiform gyrus, the preeminent area involved in face processing (Critchley et al., 2000; Grelotti et al., 2005; Pierce, Muller, Ambroses, Allen, \& Courchesne, 2001; Schultz et al., 2000), despite normal retinotopic and early visual system organization (Hadjikani et al., 2004; Pierce, Haist, Sedaghat, \& Courchesne, 2004). The neuroimaging findings are not without challenge, however: a very recent study has shown significant fusiform activation in autism, especially in the right hemisphere, as would be expected, and with greater activation in response to familiar than unfamiliar faces, as is also expected (Hadjikani et al., 2004; Pierce et al., 2004; see also, Dalton et al., 2005). The autistic individuals do show a more limited cortical network than controls in response to familiar faces in this study, but the presence of FFA activation in autism is important and contrasts with most existing studies.

The second perceptual domain, concerning the extent of global or holistic processing in autism, has also been wellstudied with many, but not all, investigations reporting that autistic individuals tend to focus more on the parts of a stimulus and to experience difficulty in deriving the global entity or whole. ${ }^{1}$ For example, autistic individuals fail to take the entire visual context into account (Happé, 1996; Ropar \& Mitchell, 1999) and fail to perceive impossible geometric figures, a task which requires part integration (Mottron \& Belleville, 1993). Using a wide variety of paradigms, investigations have also revealed that autistic individuals show enhanced detection of local targets in visual search (Plaisted, O'Riordan, \& Baron-

\footnotetext{
1 The terms global, holistic and configural are often used interchangeably in the literature and we do so here too. However, we examine possible distinctions between them in the final discussion.
}

Cohen, 1998; Plaisted, Swettenham, \& Rees, 1999), perform well on tasks such as Block Design and Object Assembly that require a local focus (Minshew, Goldstein, \& Siegel, 1997) and exhibit superior performance in detecting embedded figures (Happé, 1999; Jolliffe \& Baron-Cohen, 1997; Shah \& Frith, 1983). One recent study has revealed that autistic children use gestalt grouping heuristics significantly less often than controls do, resulting in difficulties appreciating interelement relationships (Brosnan, Scott, Fox, \& Pye, 2004). These findings are compatible with the framework, termed 'weak central coherence' (Frith, 2003; Frith \& Happé, 1994), which posits that a fundamental problem in autism is the difficulty in drawing together or integrating individual pieces of information (perceptual or conceptual) to establish meaning, with the resultant reliance on piecemeal, local information rather than on the overall context. Despite the existing evidence, the extent to which autistic subjects truly do show a local bias or do fail to derive the whole is somewhat controversial in itself and this point is specifically addressed in the experiments below.

Although there are now rich literatures focusing on the nature of face processing and the tendency to focus on local rather than global information in autism, each domain has many open questions and, moreover, there is little consideration of the relationship between these visual processes. This relationship, however, is of great interest in cognitive neuroscience. Faces form a class of perceptually similar visual stimuli and are, therefore, thought to be the paradigmatic example of a stimulus that relies heavily on configural processing, with the gestalt or holistic properties of the stimulus possibly even overriding the contribution of its individual components (Farah, Wilson, Drain, \& Tanaka, 1995; Leder \& Bruce, 2000; Maurer, Le Grand, \& Mondloch, 2002; Tanaka \& Farah, 1993; Tarr \& Cheng, 2003; Yovel, Paller, \& Levy, 2005). One might expect then that any difficulty in deriving the global configuration would substantially impair the ability to process faces. Indeed, individuals with integrative visual agnosia who experience difficulty in deriving configural information, are also impaired both at recognizing known faces and at discriminating novel faces. The reverse finding is also reported: individuals who are impaired at face processing either as a result of a brain damage (acquired prosopagnosia) (Barton, Press, Keenan, \& O'Connor, 2002; Behrmann \& Kimchi, 2003) or as a result of a congenital problem are also impaired at extracting configurations from local elements (Behrmann et al., in pressBehrmann, Avidan, Marotta, \& Kimchi, 2005; Le Grand, Mondloch, Maurer, \& Brent, 2004). A further indication of the relationship between faces and configurations comes from comparisons of performance on upright versus inverted faces, relative to objects. Typically, for normal individuals, recognition and discrimination of faces is better for upright than for inverted faces (Yin, 1969) and this difference holds to a lesser extent for objects. The disproportionate face versus object inversion effect is taken to reflect the fact that upright faces are processed globally or as a whole with extraction of the second-order 
relational features-when faces are inverted, the whole or configuration is no longer available and a more part-based system is utilized, leading to the observed decrement in performance (Farah, Tanaka, \& Drain, 1996; Maurer et al., 2002; Moscovitch \& Moscovitch, 2000). Consistent with this, prosopagnosic individuals often do not show the face inversion effect in tasks requiring face discrimination and may even do better on inverted than upright faces presumably because their part-based strategy can proceed unhampered by attempts at configural processing (Behrmann et al., 2005; Farah et al., 1996; Marotta, McKeeff, \& Behrmann, 2002).

If autistic individuals focus unduly on the local features of the input, this might adversely impact their face processing and result in a greater dependence on parts than on the whole of a face. Indeed, some studies have reported that individuals with autism rely more on individual components of the face such as the lower face or the mouth than normal individuals (Hobson et al., 1988) and there is a growing consensus that individuals with autism process faces in a more analytical, feature-based fashion (Hobson et al., 1988), and attend to different features of a face (Joseph \& Tanaka, 2003) than do their non-autistic counterparts. This bias towards local elements in autism may also interfere with the extraction of the second-order statistics, the very process thought to be critical for face recognition and discrimination and for the superior processing of upright over inverted faces (Carey \& Diamond, 1994; Rhodes, 1988). Indeed, individuals with autism are not as affected in behavioral studies by inversion of a face as their controls (Boucher \& Lewis, 1992; Davies, Bishop, Manstead, \& Tantum, 1994), show even less of a N170 ERP difference between upright and inverted faces than controls and do not show temporal or gamma band activity thought to be necessary for binding components of upright faces (Grice et al., 2001; McPartland, Dawson, Webb, Panagiotides, \& Carver, 2004). All of this is consistent with the idea that autistic individuals may proceed in a more part-based fashion and attend to local aspects of the input (although see Tantam, Monaghan, Nicholson, \& Stirling, 1989). Note, however, that some recent studies have challenged the idea that autistic individuals experience difficulty in configural face processing; for example, children with autism are subject to the Thatcher illusion, just like their control counterparts, suggesting that they can indeed perceive second-order relational features (Rouse, Donnelly, Hadwin, \& Brown, 2004). Also autistic individuals show a processing advantage for recognizing the mouth in context compared with when it is shown in isolation (Joseph $\&$ Tanaka, 2003) and, when cued, are further assisted in generating the whole-face advantage (Lopez, Donnelly, \& Hadwin, 2004), suggesting that contextual information is processed. As is evident, there remain many unanswered questions concerning configural processing, its relation to possible local preference or bias in autism and its impact on face processing.

Although the emphasis of much of the visuoperceptual work in autism has been on the processing of faces, individuals with autism have also been shown to perform poorly in recognition memory for other stimuli such as cats, horses and motorbikes (Blair, Frith, Smith, Abell, \& Cipolotti, 2001). An obvious outstanding question is whether any changes in their perceptual performance on these other classes of objects may also perhaps be explained by the trend towards local processing. Although there is consensus that faces are the paradigmatic stimulus requiring configural representation, there is growing acknowledgement that other visual stimuli might also require configural processing. For example, studies have shown that, as is true for faces, local shape and surface features may not be the most efficient strategy for the purpose of discrimination and identification of objects which belong to the same class and are perceptually similar as in the case of cars, birds, bodies, individual Greebles (see Fig. 6, for example, of this novel 3D rendered object) (Gauthier \& Tarr, 1997) or any other class of homogeneous exemplars - to efficiently differentiate individual exemplars, additional details and 'configural' or relational information may be necessary (Gauthier \& Tarr, 2002; Maurer et al., 2002; Seitz, 2002; Tarr \& Cheng, 2003). If this is so, individuals with autism who focus on local details might also experience difficulty processing other non-face stimuli when the need to do individual level differentiation arises, although the difficulty might be exaggerated for faces given the nature of the extreme homogeneity of the exemplars.

In three sets of studies, we address face processing, configural processing and the relationship between them as well as object processing more generally. We start off by examining the face processing abilities in a sample of 14 autistic adults. We then present the findings from two experiments designed to examine configural processing in the same individuals and to explore the extent to which these individuals exhibit a local bias. We also examine correlations between face and configural abilities. Finally, in the third set of experiments, we examine the perceptual performance of these individuals on other non-face visual objects, all drawn from the same stimulus class and sharing many perceptual features, to evaluate the specificity of any visuoperceptual alterations in autism.

\section{General methodology}

\subsection{Participants}

The participants were 14 high-functioning adult individuals with autism ( 12 male and 2 female), between 19 and 53 years of age, and 27 neurotypical control individuals, with 2 control subjects matched as closely as possible by gender, age and education level to each autistic individual. ${ }^{2}$ The mean full scale IQ score of the autistic group was 104.1 (S.D., 17.4). All participants had visual acuity of at least 20/40, with correction if necessary. Demographic characteristics of

\footnotetext{
2 Because one of the control subjects was well-matched to more than one autistic individual, we had 27 rather than 28 control subjects.
} 
Table 1

Biographic details and IQ scores of autistic individuals

\begin{tabular}{rllrrr}
\hline & Autistic (age/gender) & Education level & VIQ & PIQ & FSIQ \\
\hline 1 & $34 / \mathrm{F}$ & 12 & 89 & 78 & 83 \\
2 & $30 / \mathrm{M}$ & 16 & 104 & 116 & 110 \\
3 & $19 / \mathrm{M}$ & 13 & 139 & 106 & 126 \\
4 & $47 / \mathrm{M}$ & 8 & 90 & 98 & 93 \\
5 & $41 / \mathrm{F}$ & 12 & 80 & 77 & 77 \\
6 & $46 / \mathrm{M}$ & 12 & 88 & 103 & 96 \\
7 & $42 / \mathrm{M}$ & 12 & 94 & 90 & 91 \\
8 & $24 / \mathrm{M}$ & 13 & 116 & 116 & 118 \\
9 & $31 / \mathrm{M}$ & 14 & 130 & 113 & 124 \\
10 & $53 / \mathrm{M}$ & 18 & 118 & 119 & 117 \\
11 & $19 / \mathrm{M}$ & 12 & 78 & 81 & 78 \\
12 & $40 / \mathrm{M}$ & 15 & 113 & 128 & 123 \\
13 & $35 / \mathrm{M}$ & 14 & 106 & 105 & 107 \\
14 & $22 / \mathrm{M}$ & 14 & 111 & 112 & 114 \\
\hline
\end{tabular}

the autistic subjects are provided in Table 1 along with IQ scores. This study was approved by the Institutional Review Boards of the University of Pittsburgh and Carnegie Mellon University. Written informed consent was obtained from all participants.

Autistic participants had no identifiable etiology such as tuberous sclerosis or fragile-X syndrome. Screening tests to determine eligibility of the participants with autism included the Wechsler Adult Intelligence Scale-III (Wechsler, 1997), the Kaufman Test of Educational Achievement (KTEA)(Kaufman \& Kaufman, 1985), the Autism Diagnostic Observation Schedule (ADOS) (Lord et al., 1989) and the Autism Diagnostic Interview (ADI \& ADI-Revised; (Le Couteur et al., 1989; Lord, Rutter, \& Le Couteur, 1994)). The diagnosis of autism provided by the two structured instruments was confirmed by expert clinical opinion (Dr. Minshew). Subjects with autism were also required to be in good medical health, free of seizures and have a negative history of traumatic brain injury. All autistic subjects were cooperative.

Controls were volunteers recruited from the community who met preset inclusion and exclusion criteria. Potential controls were screened by completion of a questionnaire on demographic information and family and personal history. Controls were required to be in good physical health, free of regular medication usage and have good peer relationships, based on report and staff observations during testing. Controls were excluded if they had a history of neuropsychiatric disorder.

\subsection{General procedure}

The experiments were conducted on a Macintosh Powerbook 540C (9.5 in. monitor) or a Macintosh Quadra 650 (15 in. monitor) and were executed with PsyScope version 1.2.1 (Cohen, MacWhinney, Flatt, \& Provost, 1993) or with RSVP 2.5 Software (Tarr, 1994). All responses were recorded with two keys marked to identify the stimulus-response mapping either on the keyboard or, for the first few subjects, on a button box customized to run with PsyScope. Participants sat in a dimly lit room at a viewing distance of approximately $60 \mathrm{~cm}$ from the screen. All experiments were completed in a single session. Reaction time (RT) and accuracy were recorded in all experiments (except for those tasks assessing spatial frequency thresholds).

\section{Face processing}

\subsection{Gender and individual level discrimination of faces}

This first experiment was designed to examine the face processing abilities of the autistic individuals. Based on previous findings, we expect that the autistic individuals may perform more poorly than their control counterparts. A further prediction is that any observed difference will be disproportionately exaggerated as the level of categorization becomes more specific (individual as opposed to gender level) since it is at this level that the need for more fine-grained perceptual discrimination, and the reliance on second-order relations, becomes more critical. This experiment has been used successfully in the past to show this exact pattern of findings in individuals with prosopagnosia (Behrmann et al., 2005; Gauthier, Behrmann, \& Tarr, 1999).

\subsubsection{Design and procedure}

The stimuli consisted of 60 grey-scale faces (half male, half female) scanned from a 3D laser and obtained from Heinrich Bülthoff and Niko Troje (Max Planck Institute, Tübingen, Germany). All faces were cropped using the same $5.72 \times 7.62 \mathrm{~cm}$ oval window to remove cues from the hairline and face contour (see Fig. 1a). There were no diagnostic or salient cues on these faces. On each trial, two faces appeared side by side on a computer screen until the subject pressed one of two keys to respond 'same' or 'different'. The distance from the middle of each face was $10.67 \mathrm{~cm}$ and each face subtended a visual angle of $1.8^{\circ}$ horizontally and $2.5^{\circ}$ vertically. Accuracy and RT were measured. The level of categorization (perceptual similarity) was manipulated (see Fig. 1a) such that a pair of stimuli could contain faces that were (1) identical (20 trials), (2) different gender and individual (GI, 30 trials) and (3) same gender, different individual (I, 10 trials). The trials were randomized across conditions within a block.

Because autistic subjects are almost always slower than their non-autistic counterparts in producing responses and are more variable in their speed of response, examining absolute reaction times may be misleading. Furthermore, simply focusing on interactions, for example, group (autistic and control) $\times$ condition (gender and individual) with RT as the dependent measure may be subject to scaling problems that result merely from differences in baseline performance between the groups rather than from a real interaction between group and the conditions of interest. These analytical problems are well-recognized and have plagued the literature previously, for example, in the domain of schizophrenia (Miller, 
(a) Face discrimination

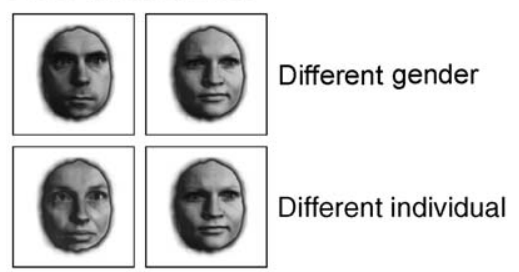

(b)

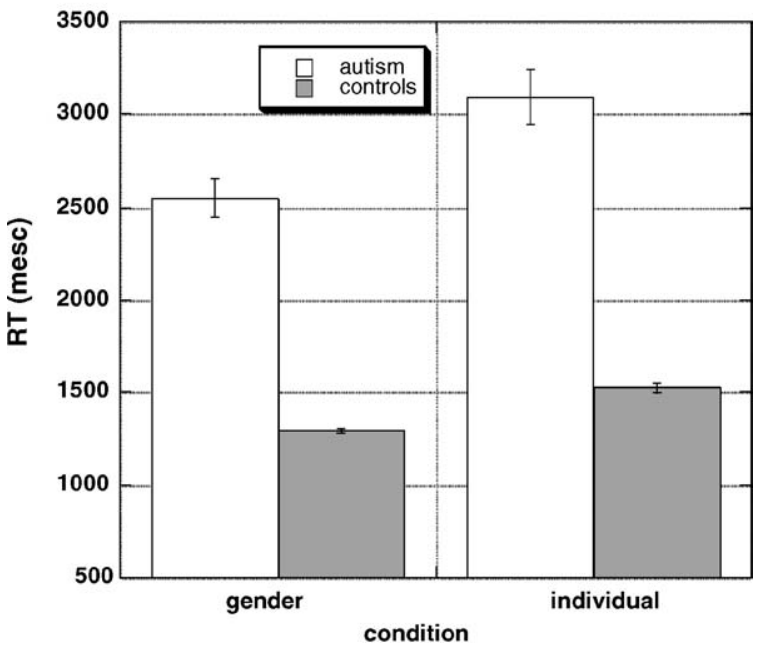

Fig. 1. Examples of stimuli and results for face experiments. (a) Examples of stimuli for face discrimination experiment, including one trial where faces differ on the basis of gender and one trial where faces differ on the basis of individual identity. (b) Mean of median RT (and one SE) for correct different trials as a function of conditions of discrimination for autistic and control groups.

Chapman, Chapman, \& Collins, 1995). In an attempt to be cognizant of these potential scaling problems, we not only use the median (to offset the influence of outliers) for each cell for each individual but also more importantly, the percentage change across the experimental conditions is calculated and compared across groups to control for differences in the absolute base RT. We also examined the RTs of each individual and ascertained whether the individual data fall outside of the 95\% confidence interval of the control subjects, following the recommendations for single case study analysis (Crawford \& Garthwaite, 2004) and other statistical recommendations (Cumming \& Finch, 2005). Accuracy is also analyzed. Note that participants are instructed to respond both quickly and accurately. It is well-known in the neuropsychological literature that, under conditions of unlimited exposure duration such as used here (non-data limited), accuracy may not be the best measure: subjects may simply spend inordinate amounts of time until they are certain that their response is correct and so RT is usually a more telling measure. Additionally, there are case reports of agnosic individuals, in whom accuracy measures suggest normal performance, but the corresponding RT data clearly indicate a marked impairment (Delvenne, Seron, Coyette, \& Rossion, 2004; Gauthier, Behrmann, et al., 1999; Gerlach, Marstrand, Habekost, \& Gade, 2004). It is for these reasons that the emphasis of the analysis here is on the
RT rather than on accuracy. We recognize that emphasizing accuracy over speed or vice versa may produce somewhat different results, a point we take up again in the final discussion.

\subsubsection{Results and discussion}

There is neither a group difference nor an interaction of group $\times$ condition in accuracy $(F<1)$, which is high in both groups (mean, S.D.: autism 92\%, 13.7\%; controls 95\%, $6.9 \%$ ) - this is not surprising given the unlimited exposure duration of the displays but warrants further discussion and is taken up again later in the paper. The RT analysis performed on the median of the correct different trials (for each condition) reveals slower RTs for autistic than normal individuals $(F(1,39)=11.21, p<0.001)$, as reflected in Fig. $1 b$. There is also a marginally significant interaction with condition $(F(1,39)=4.2, p<0.07)$ : autistic individuals are slower at individual versus gender discrimination trials than the control subjects (difference: autism $656 \mathrm{~ms}$, controls $226 \mathrm{~ms}$ ). A $t$-test based on percent RT increment in the individual over the gender trials (9.7\% controls; $14.5 \%$ autistic) yields a significant difference between groups $(t(39)=7.1, p<0.05)$, further confirming the increased difficulty for the autistic participants in the individual level discrimination. As expected, the autistic group was slower than controls but perhaps more pertinent is the difference across them when percentage RT increment is considered. Table 2 shows the number of autistic individuals whose data fall outside the $95 \%$ confidence interval of the control group in the individual level condition and, as is evident, this is so in 11 out of the 14 participants.

The group difference is not obviously attributable to differences in IQ across the autistic and control groups. We correlated the speed of correct same/different decisions made by the autistic individuals with their IQ scores and no significant correlation was noted with VIQ $(p=0.8)$, PIQ $(p=0.78)$ or FSIQ $(p=0.54)$. It is also of interest that when we compare the RT data from only those autistic subjects $(n=9)$ whose performance IQ is over 100 (the highest functioning subjects) with their corresponding controls, the autistic group is still significantly slower $(F(1,16)=4.8, p<0.05)$ and, in addition, the difference in percent RT across conditions is still evident (390 ms in controls $11.5 \%$; $1035 \mathrm{~ms}$ difference in autism 16\%).

This first experiment suggests a significant slowing in face processing in the autistic participants, relative to the controls. Of particular relevance is the relatively exaggerated difficulty for the autistic individuals on the individual level discrimination trials, which are thought to rely to a greater extent on configural processing, compared with the gender trials. Note that the group difference is apparent in RT rather than in accuracy - because the participants were not under a response deadline so as to mimic a naturalistic situation as best as possible, they may have taken longer but eventually were able to respond with high accuracy. This first experiment reveals a difference in face processing between the autistic group and the controls, and we now explore in greater detail the nature 
Table 2

Tabulation of autistic individuals falling outside of the $95 \%$ confidence intervals calculated from the normal control subjects

\begin{tabular}{|c|c|c|c|c|c|c|}
\hline & Autistic (age/gender) & Faces $^{\mathrm{a}}$ & Global local $^{\mathrm{b}}$ & Configural element $^{\mathrm{c}}$ & Objects $^{\mathrm{d}}$ & Greebles $^{\mathrm{e}}$ \\
\hline 1 & $34 / \mathrm{F}$ & - & + & + & + & + \\
\hline 2 & $30 / \mathrm{M}$ & - & + & inc. & + & + \\
\hline 3 & 19/M & + & - & - & - & - \\
\hline 4 & $47 / \mathrm{M}$ & + & + & - & - & - \\
\hline 5 & $41 / \mathrm{F}$ & + & - & + & + & + \\
\hline 6 & $46 / \mathrm{M}$ & + & + & + & + & + \\
\hline 7 & $42 / \mathrm{M}$ & + & + & + & $*$ & - \\
\hline 8 & $24 / \mathrm{M}$ & + & + & + & + & + \\
\hline 9 & $31 / \mathrm{M}$ & - & - & + & - & - \\
\hline 10 & $53 / \mathrm{M}$ & + & + & + & + & $\mathrm{n} / \mathrm{a}$ \\
\hline 11 & 19/M & + & + & + & + & $\mathrm{n} / \mathrm{a}$ \\
\hline 12 & $40 / \mathrm{M}$ & + & + & - & + & + \\
\hline 13 & $35 / \mathrm{M}$ & + & + & + & + & + \\
\hline \multirow[t]{2}{*}{14} & $22 / \mathrm{M}$ & + & + & + & + & + \\
\hline & Total & 11 & 11 & $10 / 13$ & 10 & $8 / 12$ \\
\hline
\end{tabular}

'+': Falls outside the $95 \%$ confidence intervals of the control group; '-'? falls within the $95 \%$ confidence intervals of the control group; '*': falls at the $95 \%$ confidence interval of the control group; n/a: did not perform this task; inc.: did not complete task in entirety.

a On trials of two different individual faces of the same gender.

b Calculated as $\{$ (global inconsistent - global consistent $) /($ local inconsistent - local consistent $)\}$.

c Calculated as (CS-ES) for all prime durations many element trials.

d On trials consisting of two different exemplars (e.g., two different chairs).

e On trials consisting of two different individual Greebles; only 12 autistic subjects performed this task.

and extent of the configural processing abilities in these same individuals.

\section{Configural processing in autism}

Although the weak central coherence hypothesis suggests that autistic individuals may exhibit difficulty in integrating information into a coherent or meaningful whole from local parts (both perceptually and conceptually as in low and high level weak central coherence), the empirical findings are more complicated. While some studies have shown that autistic individuals are better able to identify the local than global letters in compound stimuli and to focus on local elements (sometimes to an even greater degree than is true for the control subjects) in visual search (Plaisted, 2000; Plaisted et al., 1998), this is not always the case. For example Mottron and his colleagues (Mottron, Burack, Stauder, \& Robaey, 1999; Mottron, Burack, Iarocci, Belleville, \& Enns, 2003) have reported that autistic individuals do not differ from non-autistic subjects in deriving the identity of the global letter of compound stimuli (although perhaps even more surprising is the absence of the expected global advantage for normal individuals in some of these studies). The presence of a global bias in autism has also been observed in other studies using somewhat different displays (Ozonoff, Strayer, McMahon, \& Filloux, 1994; Rinehart, Bradshaw, Moss, Brereton, $\&$ Tonge, 2000). Note that there are two separate issues: the first concerns the possible enhanced local bias in autism and the second concerns the ability to derive a global configuration. These may be somewhat independent and separable and we explore both of them in the following experiments. The first experiment uses the well-known Navon (1977) stimuli in which letters are identified at the global or local level and the second experiment uses a primed matching paradigm, which allows for a more fine-grained and temporally extended analysis of configural processing.

\subsection{Global/local processing with compound letter stimuli}

This experiment adopts the well-known compound stimuli, which are large hierarchical letters made up of small letters, in which the identity of the local letters is either consistent or inconsistent with that of the global letter (see Fig. 2a). In the version of the task we used, in separate blocks of trials, subjects identify the letter, via key press ('s' or ' $h$ '), at either the local or the global level. All else being equal, in normal subjects, the global letter is identified faster than the local letter, and conflicting information between the global and the local levels exerts asymmetrical global-to-local interference (Navon, 1977). We note that many parameters affect these findings including the length of the exposure duration (Navon, 1977; Paquet \& Merikle, 1984), sparsity of local letters (Martin, 1979), foveal placement of the stimulus (Pomerantz, 1983) and spatial certainty (Lamb \& Robertson, 1988; Navon, 2003). In addition, the blocked version of the task used here requires focused attention at either the global or local level rather than divided attention in which, within a block, identification can occur at either level. Whether the paradigm is run blocked or mixed appears to affect autistic individuals differentially (Plaisted et al., 1999).

\subsubsection{Design and procedure}

The stimuli were four hierarchical letters of two types: consistent letters, in which the global and the local letters 
(a)

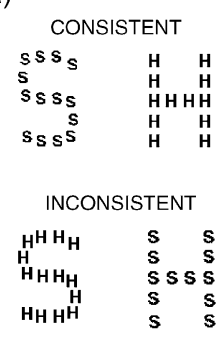

(b)

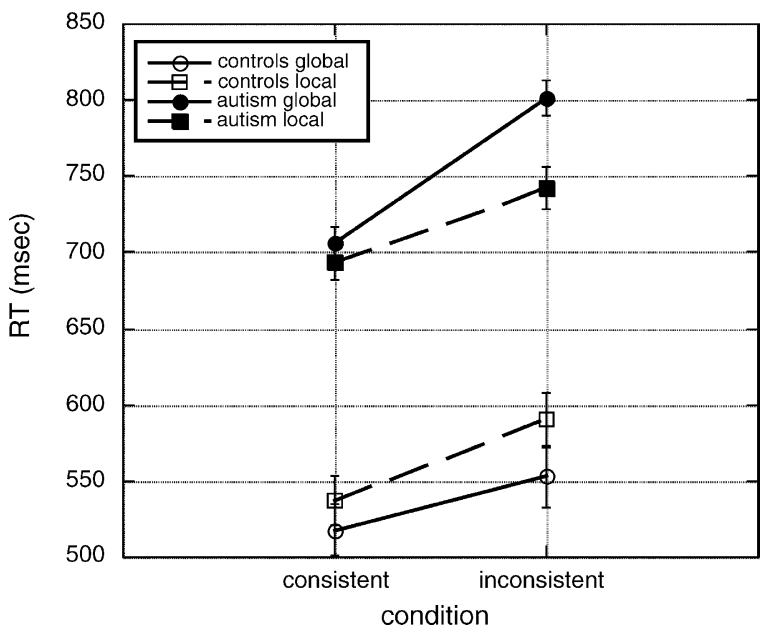

Fig. 2. Examples of stimuli and results of global/local task. (a) Four compound stimuli, two of which are consistent and share identity at the global and local level and two of which do not share identity at the global and local level. (b) RT (and one SE) for means for control and autism group for global and local identification as a function of consistency.

shared identity (a large $\mathrm{H}$ made of smaller $\mathrm{Hs}$ and a large $\mathrm{S}$ made of small Ss) or inconsistent letters, in which the letters at the two levels had different identities (a large $\mathrm{H}$ made of small Ss and a large S made of small Hs; see Fig. 2a). The global letter subtended $3.2^{\circ}$ in height and $2.3^{\circ}$ in width, and the local letter subtended $0.44^{\circ}$ in height and $0.53^{\circ}$ in width.

The experiment consisted of the factorial combination of two variables in a repeated measures design: globality (global identification versus local identification), and consistency (consistent stimuli versus inconsistent stimuli). The two tasks, local or global identification, were administered in separate blocks of 96 experimental trials each, preceded by 10 practice trials. The consistent and inconsistent letters were randomized within block with each letter occurring on an equal number of trials, for a total of 192 trials. Before each block, participants were verbally instructed to respond to the global or local letters. Each trial was initiated with a central fixation cross of $500 \mathrm{~ms}$ duration. This was immediately replaced by one of the four possible stimuli, which remained centrally on the screen until a response was made. Participants were instructed to press the left key on the button box (or keyboard) to indicate a response of ' $s$ ' or the right key for ' $h$ '. The order of the blocks and response designation was counterbalanced across subjects.

\subsubsection{Results and discussion}

At the outset, we note that there is neither a group difference between autistic and control subjects, nor an interaction of any sort, in the accuracy data (all $F<1$ ). Autistic and control subjects were correct on average $98 \%$ (S.D., 2\%) and $98.2 \%$ (S.D., 1.3\%) of the time, respectively. The high accuracy rate is not surprising given the unlimited exposure duration and ease of task (making s/h decisions). The RT data, calculated on the median for each subject for each condition, reveals a significant three-way interaction between group $\times$ globality (global and local) $\times$ consistency (consistent and inconsistent $)(F(1,39)=4.9, p<0.05)$. There are also main effects of group and of globality $(p<0.0001)$. As is evident from Fig. 2b, under these testing conditions, the control subjects responded quickly and showed a slight advantage for global over local identification (23 ms) and a slight asymmetry with greater slowing in the inconsistent case (relative to the congruent case) when local identification is required (interference from globally incongruous letter) than when global identification is required (interference from locally incongruous letters). This global advantage and the globalto-local interference replicates the standard findings (Navon, 2003), although the condition differences may not be as large as usual given the unlimited duration and repeated foveal presentation.

The autistic subjects were slower than the control subjects overall, but most importantly, a different pattern of performance is observed for them (Fig. 2b). The autistic group is overall faster for local than global identification although this difference comes from the inconsistent trials: there is no statistically significant difference between global and local identification in the consistent case, but in the inconsistent case, local identification is faster than global identification $(p<0.05)$. The latter result, namely greater slowing in the inconsistent case when global identification is required, indicates a large local-to-global interference. The faster local identification and the local-to-global interference both indicate that autistic individuals show a local bias in their processing. It is the case, however, that there is no obvious local advantage in the consistent case suggesting that there may be some partial processing of the global identity too which, when congruent with the local letter, can be extracted. We can infer then that, under the conditions employed here, the autistic individuals were able to derive the global configuration in the consistent condition, but that their local bias gave rise to large interference in the inconsistent condition, suggesting that it was difficult to attain a stable global configuration when the elements had a conflicting identity.

Given the ongoing controversy in the autism literature concerning the extent to which processing is locally biased and the extent to which configural processing is possible, and given the suggestion that the autistic group in this experiment may be able to derive the global identity as well as the local identity in the consistent case, we examined the data of each autistic subject individually. To this end, we calculated the number of autistic individuals who fall outside 
the $95 \%$ confidence intervals of the control group, calculated as $\{$ (global inconsistent - global consistent)/(local inconsistent - local consistent) $\}$ and 11 of the autistic subjects fall outside the intervals. We also conducted pairwise ANOVAs with each autistic subject and his/her two matched controls as the between-subjects variable and globality and consistency as the within-subjects variables. Nine of the 14 pairwise comparisons show the significant interaction with group, suggesting that the pattern we observe for the group is present in the majority of autistic subjects but not in all of them as in the confidence interval analysis.

A number of possible explanations for the observed variance within the autistic group may exist. One immediate possibility is that autistic individuals really do differ in the extent to which they can undertake configural processing. Another possibility is that 'weak coherence' is a cognitive style rather than a deficit per se and the extent to which configural processing manifests is a function of the autistic individual ability to avoid/adopt this style when instructed to do so (Booth, Charlton, Hughes, \& Happé, 2003). An alternative explanation has to do with the parameters of this experiment: because the stimuli are presented for unlimited exposure duration, with enough time, many autistic subjects may be able to derive the global shape. It is also the case that the presence of multiple local elements may assist this process; a display that is relatively sparse with few local elements biases away from global shape whereas the presence of multiple local elements (as in this case) assists in the extraction of the whole (Bacon \& Egeth, 1991; Kimchi, 1998). Thus, in these conditions, with enough time and enough local elements, autistic subjects may be able to assemble the global shape (especially with help from the consistent local elements). Whether they can still derive the global shape under more taxing conditions remains to be determined.

\subsection{Microgenetic analysis of the perceptual organization of hierarchical stimuli}

Given the uncertainty about whether autistic subjects can derive global identity under more challenging conditions and the apparent within-group variance, this next experiment probes the configural processing of the autistic subjects in greater detail. In this experiment, we varied the number of local elements across two displays, one of which contained a few large elements and one of which contained many small elements. The former is thought to bias local processing but the latter is thought to bias global processing (Kimchi, 1998, 2000). Importantly, as well as using displays that have few large or many small elements, a prime is presented and the temporal interval between the appearance of the prime and the probe display is manipulated. This approach, referred to as the microgenetic approach, involves examining the temporal evolution of the percept, rather than just the final outcome of perception. One prediction is that, with enough time, autistic individuals are able to derive the global whole but cannot do so under brief exposure durations. The time course may also interact with the number of elements such that with sufficient time only the many- but not the few-element condition may lend itself more easily to configural processing in autism (as in the previous experiment).

This experiment used the primed matching paradigm (Beller, 1971): participants view a prime followed immediately by a pair of test figures, and judge, as rapidly and accurately as possible, whether the two test figures are the same or different (see Fig. 3a). No response is made to the prime itself. Primes and probes are made of few or of many elements (but in a single trial, prime and probe always share the same number of elements). There were two types of test pairs defined by their similarity to the prime (see Fig. 3a): the element-similarity (ES) test pairs in which the test figures were similar to the prime in their local elements but differed in global configuration, and the configuration-similarity (CS) test pairs in which the figures were similar to the prime in global configuration but differed in local elements. The speed of same responses to the test figures depends on the representational similarity between the prime and the test figures: responses are faster when the test figures are similar to the prime than when they are dissimilar to it. For example, it is now well-known that, in neurotypical individuals (Behrmann \& Kimchi, 2003; Kimchi, 1998), the availability of elements and configuration depends on the number and relative size of the elements. For few, relatively large elements, the component elements are available early on in processing (ES advantage) and, only, with more time, are they configured or grouped into a global configuration. For manyelement patterns, there is an interesting U-shaped function: the configuration is available very early (CS advantage), even at $40 \mathrm{~ms}$, suggesting that normal individuals derive the forest rapidly before the trees. At the intermediate durations, the elements themselves are individuated and finally, at long durations $(690 \mathrm{~ms})$, both the configuration and the elements are available to the observer. By varying the timing of the prime in relation to the probe, we can tap earlier and later internal representations (Behrmann \& Kimchi, 2003; Kimchi, 1998, 2000; Sekuler \& Palmer, 1992). The question addressed here is whether the same effects of number and relative size of elements influence the perception of the autistic individuals, as normal individuals, and if so, how early in time.

\subsubsection{Design and procedure}

Responses to the visual displays were made by pressing one of two response keys, and RTs and accuracy were recorded by the computer. Participants used their dominant hand for responding. The priming stimuli were few-element and many-element hierarchical patterns (global diamonds made up of circles). The few-element prime was a diamond made of four relatively large circles, and the many-element prime was a diamond made of sixteen relatively small circles. Each test stimulus consisted of two hierarchical patterns. There were two types of test pairs, ES and CS pairs, each of which gave rise to same and different responses. The global diamond subtended $1.25^{\circ}$, and the global square $0.96^{\circ}$. 
(a) Microgenesis stimuli

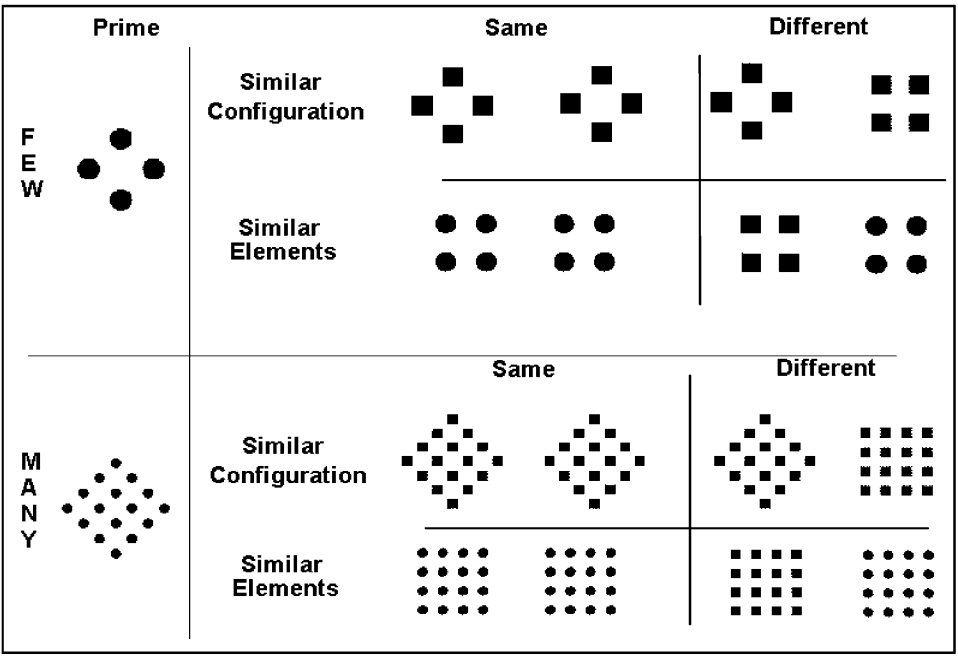

(b) Group means

Few elements Many elements

(i) Controls

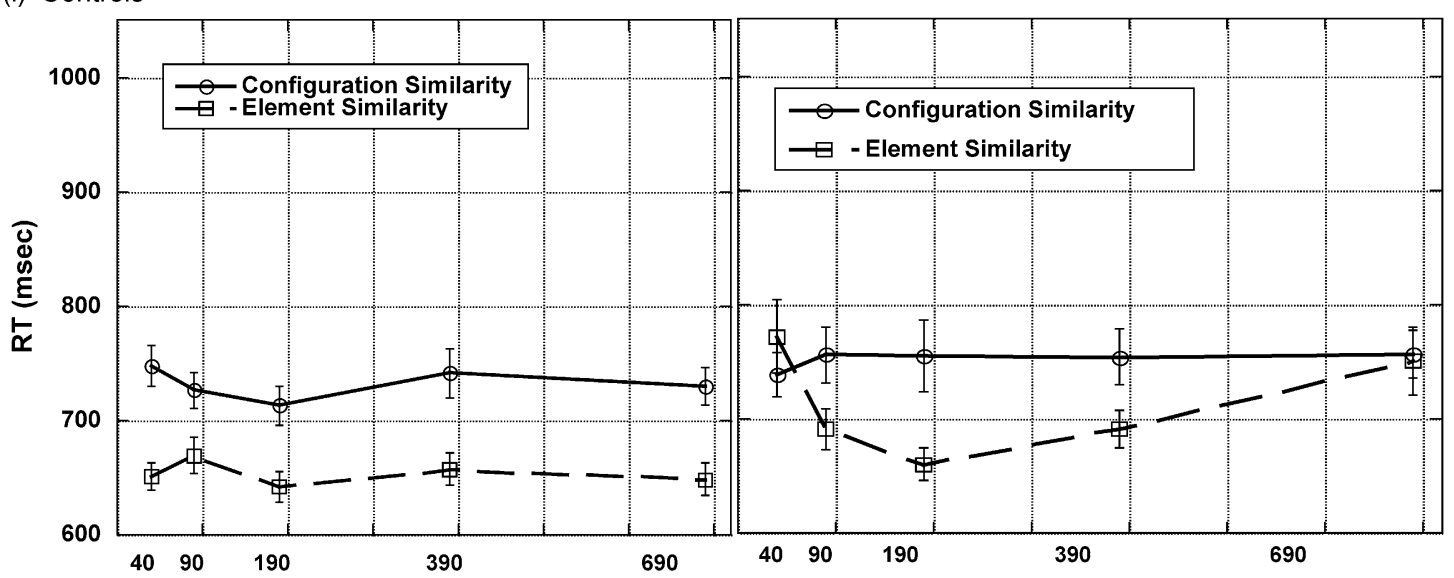

(ii) Autism

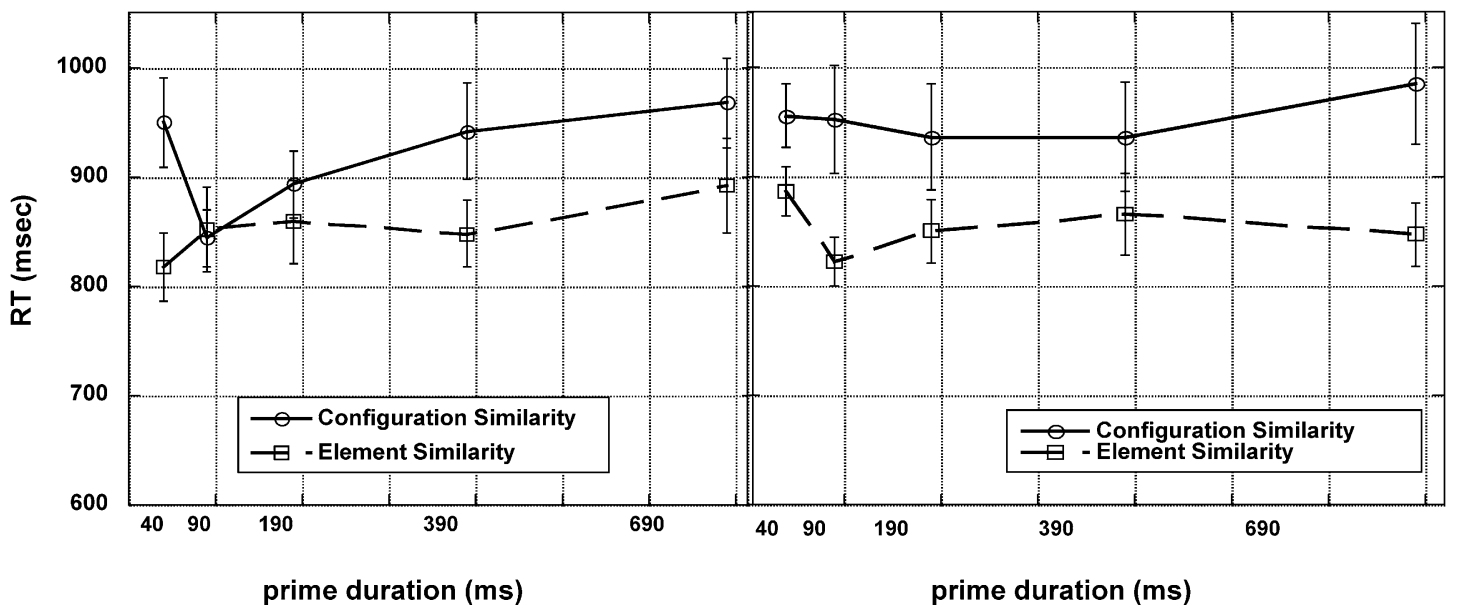

Fig. 3. Examples of stimuli and results of few/many microgenetic experiment. (a) Primed match paradigm: probes, consisting of few and many elements, are followed, after varying SOAs, by test pairs which require 'same' or 'different' responses and which are similar to the prime in elements or configuration. (b) Group means of RT (and one SE) for (i) controls and (ii) autistic individuals for few and many displays, shown for configuration similarity (CS) and element similarity (ES) trials as a function of SOA. 
Each individual circle element subtended $0.36^{\circ}$ (in diameter) in the few-element patterns, and $0.18^{\circ}$ in the many-element patterns. Each individual square element subtended $0.38^{\circ}$ in the few patterns, and $0.19^{\circ}$ in the many-element patterns. The distance between the centers of the two stimuli in a test pair was $0.7 \mathrm{~cm}$.

The experiment consisted of the factorial combination of four factors: prime type (few-element or many-element); prime duration $(40,90,190,390$ or $690 \mathrm{~ms})$; test type (ES and CS); and response choice ("same" or "different"). The few-element and many-element primes were administered in separate blocks of 160 trials each. All the combinations of the three factors (prime duration, test type and response) were randomized within block with each combination occurring on an equal number of trials. Each trial consisted of the following: first, a small fixation dot appeared in the center of the screen for $250 \mathrm{~ms}$, followed by a prime. The presentation time for the priming stimulus was equally and randomly distributed among 40, 90, 190, 390 and $690 \mathrm{~ms}$. Immediately after the presentation of the prime, the test display appeared and remained until the response, for a maximum of $3000 \mathrm{~ms}$. The test display contained two figures presented on either side of the location previously occupied by the prime. Participants had to decide whether the two figures were the same or different and respond accurately and quickly using the response keys. Each individual completed 320 trials. Sixteen practice trials were completed for each of few-element and many-element patterns before the experimental trials. We note that one autistic individual (subject 2 Table 1) and his controls were not included in this analysis as his data were incomplete.

\subsubsection{Results and discussion}

Accuracy rates were high for both groups (mean and S.D.: autism $98.3 \%$, S.D. 3.1\%; controls $98.1 \%$, S.D. $4.3 \%$ ), again not surprising given that the test pair remains on the screen for unlimited duration. There were no differences between the groups nor any interactions between group and any of the other variables on accuracy $(F<1)$. In light of this, we turn to the RT data. Mean of median correct same RTs for prime-test similarity (ES and CS) are plotted in Fig. 3b as a function of prime duration for each prime type (few-element and manyelement patterns) for the control and autistic groups (panels i and ii, respectively). Note that only same trials are analyzed as the relationship between the probe and test pair is only fully controlled when the two items in the test pair are the same (for example, prime is diamond made of circles and test pair is made of two squares, both of which are made of circles so, in both halves of the test pair, the local elements but not global configuration are shared with prime). Most importantly, as shown in Fig. 3b, the patterns of data for the two groups are statistically different. For the control group, for the few-element display (i, left panel), there is a $72 \mathrm{~ms}$ advantage for ES over CS, collapsed across duration. In contrast, for the many-element display, very early on, as well as at the longest SOA, there are equally rapid RTs for ES and CS. Only at the mid-durations is an ES advantage apparent. These data replicate the previous findings using this paradigm (Behrmann \& Kimchi, 2003; Kimchi, 1998), and other previous results showing global representation with many-element stimuli under short exposures (Navon, 1977; Paquet \& Merikle, 1984). Of particular pertinence for the comparison with the autistic group, we point out the advantage for greater priming of the local elements for few-element displays and the rapid ability to derive a global configuration of many items at early SOAs.

For the autistic group, there is also a large advantage for the ES over CS test pairs for few-element trials ( $88 \mathrm{~ms}$ ), suggesting a slightly greater local bias for the autistic subjects with few, large elements than for the controls (note the unexplained anomalous drop in CS for autistic group at $90 \mathrm{~ms}$ which is also diminishing the full extent of the local advantage or ES). Of relevance too, is the absence of priming of the configural representation for the autistic group at any prime duration whereas $\mathrm{CS}=\mathrm{ES}$ for the controls at both short and long durations for many elements; in contrast, there is a substantial advantage $(68 \mathrm{~ms})$ for ES over CS for many-element displays across all exposure durations. The major finding, then, is that there is no evidence of benefit from the prime for the CS condition in autism for many-element items as there is in normal control individuals. Instead, a robust and persistent benefit (except for $90 \mathrm{~ms}$ few elements) for the ES test pairs is obtained for few-element and many-element displays.

The statistical analyses support these findings: the difference between CS and ES is significant across the groups and as a function of few/many elements $(F(1,36)=4.6, p<0.05)$, as described above. There is also a significant interaction of test type $\times$ group $\times$ prime duration $(F(4,144)=2.7, p<0.05)$ and a marginally significant interaction of prime type $\times$ prime duration $\times$ group $(F(4,144)=1.9, p=0.09)$. Post hoc tests indicate that, at $40 \mathrm{~ms}$ SOA, there is no pairwise difference between CS and ES for the control group for the many element display whereas this difference persists for the autism group. One important point is that $40 \mathrm{~ms}$ may be too rapid for the autistic subjects to show the early configuration priming, given that there is a reduction in their speed of processing. However, there is no time point (in the range we tested) at which the configuration priming is observed for the autistic individuals suggesting that they are not exhibiting merely a temporal delay or offset relative to the controls but, rather, are showing a different pattern of behavior. As is evident from Table 2, 10 of the 13 autistic individuals who completed the experiment fall outside the normal confidence intervals on many element items (calculating all CS - ES).

The findings from this experiment are roughly compatible with those from the global/local hierarchical experiment (Section 4.1). In both cases, for the control subjects, we see evidence of global or configural processing: a small but robust advantage for global over local identification and global-tolocal interference, and an equivalence in RT for CS over ES in many-element trials in this experiment at early and late exposure durations. The autistic subjects show some access to global identity in the global/local experiment (Section 4.1), 
possibly afforded by the identity and numerosity of the local elements. In this current experiment, there is no apparent evidence for global processing and the enhancement of local processing is striking, as evident in the persistent and large difference between CS and ES in both the few and the many-element trials. To the extent that adults with autism have the capacity to derive a global whole, this ability is rather weak and possibly supported by the opportunity for local elements to prime the global shape in the global/local experiment. Note that the patterns used here are all inconsistent (squares made of circles or diamonds made of squares) so there is no such opportunity for local facilitation. The findings from this experiment are consistent with the recent data showing that autistic individuals fail to process inter-element relationships and show a bias away from gestalt grouping principles (Brosnan et al., 2004).

\subsection{Spatial frequency thresholds}

Before claiming that the reduced pattern of a global advantage in autism is one of impaired configural processing, we need to rule out some alternative explanations. One such explanation concerns possible differences in low-level spatial frequency analyses. Several researchers have suggested an involvement of spatial filters, based on spatial frequency channels, operating at early visual processing (Ginsburg, 1986) in the perception of global and local structures. For example, no latency advantage for global over local processing is found when low-spatial frequencies are removed from hierarchical stimuli (Badcock, Whitworth, Badcock, \& Lovegrove, 1990; Hughes, Fendrich, \& Reuter-Lorenz, 1990; Lamb \& Yund, 1993; Shulman \& Wilson, 1987; Shulman, Sullivan, Gish, \& Sakoda, 1986), suggesting that the global advantage effect is mediated by low spatial frequency channels. Thus, one possible explanation for the autistic subjects' reduced ability to perceive the global form of a hierarchical stimulus might concern a fundamental limitation in processing low spatial frequency information. If so, then we might expect that autistic individuals should be relatively impaired at processing low frequency displays, resulting in an increased low spatial frequency threshold, relative to their control subjects. To evaluate this, we established thresholds for the autistic subjects across a wide range of spatial frequencies and compared them to those of control participants.

\subsubsection{Design and procedure}

To document the spatial frequency function, we established, for each individual, the log contrast thresholds at 0.3, $1,3,10$ and 30 cycles per inch (cpi) using a Matlab function which implements a discrimination task. Subjects completed five blocks of trials, with 20 trials each and using displays corresponding to one of the cpis (examples of stimuli at 1 and 30 cpis are shown in Fig. 4). In each trial, a fixation point appeared on the screen for $1 \mathrm{~s}$. After $200 \mathrm{~ms}$, one image appeared for $200 \mathrm{~ms}$ followed by a blank screen for a further $200 \mathrm{~ms}$. A second image then appeared for $200 \mathrm{~ms}$ and
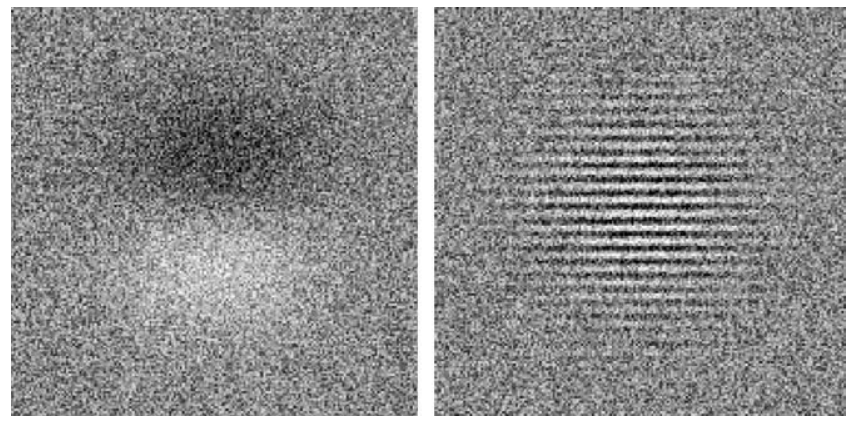

Fig. 4. Examples of stimuli for testing low-level vision. Examples of stimuli used to establish contrast thresholds across different spatial frequencies. Examples here are 1 cycle per inch (left) and 30 cycles per inch (right). In a sequential paired task, subjects indicate whether the first or second stimulus in the pair contains the grating.

it, in turn, was replaced by a $200 \mathrm{~ms}$ blank screen. At this point, the subject was required to decide whether the first or second image contained the grating. Feedback was provided to the subject after each trial. A series of five practice trials was presented before the first block and, for most subjects, the order of blocks was counterbalanced. If the response was correct, a more difficult discrimination (decreased contrast by 0.2 ) was presented on the next trial. If the response was incorrect, the contrast was increased by 0.2 . A log contrast threshold was determined for each cpi using method of limits where threshold is defined as the value of contrast that produces $82 \%$ accuracy. Note that one autistic subject was unwilling to complete this experiment and two of them completed all cpis except for 0.3 (as they completed 0.1 instead in error).

\subsubsection{Results and discussion}

The mean log contrast thresholds obtained for the autistic patients was compared with the mean of the normal subjects. A group $\times$ spatial frequency analysis reveals no difference across groups, nor an interaction of group $\times$ frequency (both $F<1$ ). This finding confirms that the autistic individuals perform within the normal boundaries in detecting low and high frequency gratings. Having ruled out the possibility that the discrepancy between the patients and the normal control subjects in their perception of the hierarchical stimuli is due to differential limitations in analyzing spatial frequency information, it seems that the failure to derive a global whole reflects a difficulty in configural processing. In the final experiments, then, we examine the impact of configural processing on other visual, non-face stimuli.

\section{Discrimination of non-face objects}

The prediction to be tested here is that the autistic subjects, who show a bias for local processing and less efficient global processing than the controls, may also perform less well than the controls on non-face objects especially as the difficulty of perceptual discrimination increases. 


\subsection{Object processing}

\subsubsection{Design and procedure}

A set of 80 grey-scale objects was created by rendering 3D object models using Silicon Graphics Inventor Software. The object models were obtained from multiple sources, including public domain sites and commercial CD-ROMS. As was the case for the face testing, on each trial, two stimuli (of varying levels of similarity) were placed side by side on a computer screen for an unlimited duration (see Fig. 5a) until the subject pressed a same or different key. Accuracy and RT were measured. The level of categorization was implemented as follows: pairs of stimuli for each of four conditions were created in the following way: (1) identical (40 trials, 2 repetitions), (2) pair where stimuli differ at basic, subordinate and exemplar levels (BSE, 20 trials: car and a duck), (3) pair where stimuli differ at subordinate and exemplar levels (SE, 20 trials): duck and a pelican) and (4) pair where stimuli are different exemplars but from the same subordinate level (E, 20 trials: 2 different ducks). The trials were randomized across conditions within a block.

\subsubsection{Results and discussion}

The analysis of the median RT on correct different trials reveals a significant interaction between group and condition $(F(2,78)=5.1, p<0.008)$; although both groups exhibit slower RTs as level of categorization becomes more specific, this is so to a somewhat greater degree for the autistic than for the control group (see Fig. 5b). Thus, autistic subjects require an additional $149 \mathrm{~ms}$ to make the subordinate over the basic (a)

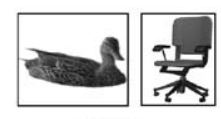

Basic
Common Objects

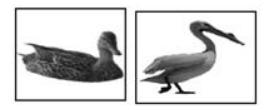

Subordinate

(b)

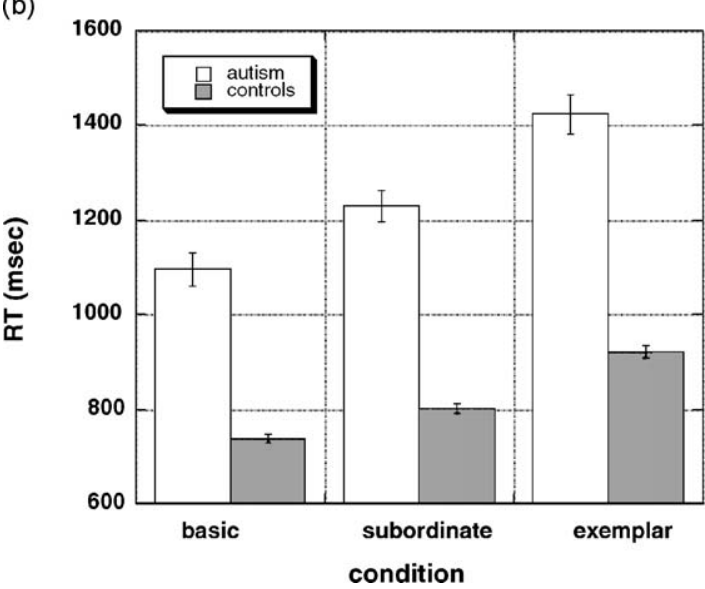

Fig. 5. Examples of stimuli and results of common object discrimination experiment. (a) Examples of stimuli from common object discrimination task, showing pairs of stimuli that differ at the basic, subordinate or exemplar levels. (b) RT (and one SE) for means for control and autism group as a function of condition of discrimination.
(6.9\% increment) judgment and a further $206 \mathrm{~ms}(10 \%)$ to make the exemplar decision, and the corresponding numbers for the control group are $62 \mathrm{~ms}(4.3 \%)$ and $122 \mathrm{~ms}(7.5 \%)$. Note that, although a significant group $\times$ condition interaction is present, the speed of the base reaction time and the magnitudes of the cross-condition difference are lower than in the face discrimination experiment (compare with Fig. 1 and note $y$-axis differences). The analysis of the percent RT difference scores using a $t$-test shows a group difference in RT between basic and subordinate $(p<0.05)$ although this does not reach statistical significance between subordinate and exemplar $(p>.05)$. Note that the between group differences do hold when a subanalysis of RT is done comparing the nine highest IQ (performance IQ > 100) autistic individuals and their controls counterparts $(p=0.043)$. Also, as shown in Table 2, 10 (and 1 on boundary) autistic participants fell outside the $95 \%$ confidence intervals of the control subjects based on RTs on the exemplar trials of the task. There was neither a group nor a group $\times$ condition interaction in the accuracy data $(F<1)$ (mean S.D.: autistic $98 \%, 3.9 \%$; controls $97 \%, 4 \%$ ), presumably because the exposure duration was unlimited and the effects manifested in speed rather than in accuracy.

\subsection{Greeble processing}

\subsubsection{Design and procedure}

The stimuli consisted of 60 grey-scale pictures of Greebles (examples in Fig. 6a). As was the case for the face and object testing, on each trial, two stimuli were placed side by side on (a)

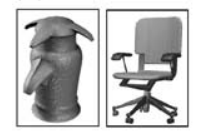

Basic
Novel objects: Greebles

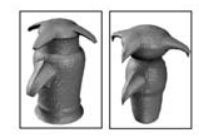

Family

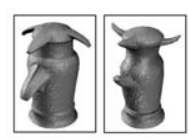

Gender

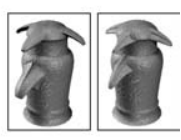

Individual

(b)

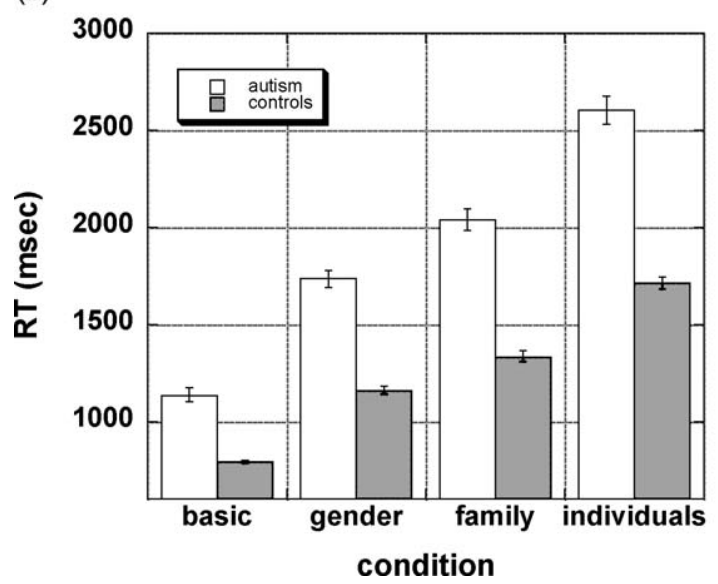

Fig. 6. Examples of stimuli and results of Greeble discrimination experiment. (a) Examples of stimuli from Greeble task, showing pairs that differ at the basic, family, gender and individual level. (b) RT (and one SE) for means for control and autism group as a function of condition of discrimination. 
a computer screen for an unlimited duration until the subject pressed a same or different key. Accuracy and RT were measured. Greebles are a class of novel stimuli, designed to mimic the processing demands of faces, i.e., all have the same number and rough geometry of local parts requiring the derivation of spatial relationships between them. Additionally, to parallel the level of categorization of faces (face versus object, two faces of different gender or individual faces of the same gender), the Greebles are designed to fall into families which all share the same main body. Within each family, there are two possible genders (appendages go up or down) and then within the family and gender, there are individual, unique Greebles. In this task, the level of categorization was implemented in the following way: there were five conditions (1) identical (63 trials), (2) basic (paired with a familiar object, such as a car), (3) different gender (G; paired with a Greeble from another gender but same family), (4) different family (F; paired with a Greeble of different family but same gender) and (5) individual (I, paired with a Greeble with different individual identity but from same family and gender). Conditions $2-5$ had 30 trials each. Only 12 autistic subjects and corresponding control subjects completed this experiment (the remaining two inadvertently completed a more complex version which included upright and inverted Greebles-we note, however, that the findings from these two subjects are roughly the same as for those tested here).

\subsubsection{Results and discussion}

An analysis of the median RT data from correct different trials reveals an interaction of group $\times$ condition $(F(3,90)=6.3, p<0.001)$. As is evident from Fig. 6b, the control subjects show a graded effect with RT increasing with difficulty of perceptual discrimination. This slowing, however, is disproportionately increased in the autism group. The difference between some conditions is almost double for the autistic than for the control groups; the difference between basic and gender is $579 \mathrm{~ms}(22 \%)$ for autistic and $319 \mathrm{~ms}$ $(17 \%)$ for controls, the difference between gender and family trials is $188 \mathrm{~ms}(5.4 \%)$ for autistic and $113 \mathrm{~ms}(4.9 \%)$ for controls and, finally, the difference between family and individuals is $631 \mathrm{~ms}$ (14.8\%) for autistic and $382 \mathrm{~ms}(13.2 \%)$ for controls. The percent difference score reveals a significant group difference in going from basic to gender $(p<0.05)$ but not between the other conditions. An analysis of the data including the highest IQ autistic individuals (only eight rather than nine as one of the high IQ autistic subjects performed the other version of the task) does reveal a group $\times$ condition interaction $(p<0.038)$ and, with the exception of family to gender, all cross-condition differences are significant. As seen in Table 2, 8 of 12 autistic individuals who completed this task fell outside the 95\% confidence intervals on the Greeble individual task. There was neither a main effect of group nor an interaction evident in the accuracy data $(F<1)$ (mean S.D.: autistics $92 \%, 13.5 \%$; controls $94.6 \%, 4.5 \%$ ).

These findings suggest that individuals with autism are slower in making decisions about Greebles compared with their control counterparts but that the nature of the decision is not equal across all conditions. For example, autistic individuals, like the controls, do not show much of a RT difference between decisions involving family (main body) and decisions involving gender (appendage direction). The autistic individuals, however, as a group, are slowed in differentiating between two Greebles compared with differentiating between a Greeble and another object (basic level) even when absolute reaction time is taken into account. Although we expected that the autistic individuals would also be disproportionately impaired in discriminating between two individual Greebles, the autistic subjects are only minimally slower than the controls when a difference score is computed and baseline RT taken into account.

\section{Relationship between configural and face and object processing}

The findings thus far indicate that the group of autistic individuals was slower at face processing than their controls, especially as the level of categorization and perceptual similarity became more fine-grained. The autistic group also showed a greater local bias than the control group and, under the testing conditions employed, did not show the configural or global processing observed in the control pattern. The autistic individuals also performed more slowly than the control group on non-face common and novel objects, and this too was true to a somewhat greater degree as the need for more precise discrimination (based on configural knowledge) was required.

Given that one of the goals of this study was to examine the relationship between face/object processing and configural processing, we performed correlation analyses first between the two configural tasks and then between them and the face/object tasks. We took the individual values from the configural tasks, which we used to establish whether the individual subjects fell outside the normal confidence intervals (see Table 2) and correlated them. The $r^{2}$ value was 0.23 $(p=0.09)$, which is encouraging given the small number of subjects and suggests some replicability of the findings across the two configural tasks. We then went on to investigate the correlation with faces/objects using the value for each subject from the global/local task.

The median RT for face processing, collapsed across condition, correlated with the configural value described above, yielded a significant $r^{2}$ value of $0.61(p=0.03)$. Although correlation is not causation, the relationship between RT in face processing and a local bias is clear in the autistic individuals. Of interest also is that a significant correlation is also observed between the effect of globality, as above, and the median RT on the Greeble experiment in the autistic group $\left[r^{2}\right.$ value of $0.47(p=0.01 ; 12$ individuals $\left.)\right]$. This same correlation does not reach significance for objects $\left[r^{2}\right.$ value of $0.21, p=0.1]$.

Taken together, the results of the correlation analyses are informative: the slowing in face (and Greeble) processing is 
well correlated with the relative superiority of processing local information. The lack of a significant correlation with objects is consistent with the idea that the objects may well rely on configural processing (especially at an individual level) but to a lesser extent and indeed, the relationship between global and object processing is much weaker.

Before turning to the final discussion, we examine the profile of the individual autistic subjects across the five experiments using the data from Table 2 which shows whether on each task, the subjects performance fell beyond the normal $95 \%$ confidence interval. Four of the 14 subjects fall outside the normal confidence intervals on all five experiments and an additional two who did not complete the Greebles task, did so on the remaining four experiments (and, as mentioned, showed the same findings as we see here on the Greeble task they did perform). A further three subjects fall below the lowest confidence intervals of the normal limits on four of the five tasks. Two subjects did so on a single task and one subject did so on two tasks (faces and global/local).

Taken together, these explorations of the patterns of individual data provide reasonably, although not perfectly, good support for the relationship between the configural and face task and to a somewhat lesser degree between configural and object and Greebles task. It is also the case that many, although not all, autistic individuals exhibit the same pattern of performance in RT.

\section{General discussion}

To date, much research in visuoperceptual processing in autism has focused on two lines of investigation. The one line of investigation has shown that autistic individuals perform poorly on tasks requiring face processing, including identity recognition as well as discrimination of gender, gaze direction and emotion (Teunisse \& De Gelder, 2003). The second line of investigation has been concerned with the extent to which autistic individuals are able to derive a coherent or meaningful whole from the local elements present in visual input. While most studies report that autistic individuals show a local bias, sometimes leading to superior performance than control subjects (Caron, Mottron, Rainville, \& Chouinard, 2004; Plaisted, Saksida, Alcantara, \& Weisblatt, 2003), the extent to which autistic individuals can integrate the local components and derive the global configuration is less clear. The focus of the current paper is on each of these lines of investigation as well as on the relationship between them. A final issue addressed is whether autistic subjects differ from their controls on non-face stimuli, so as to ascertain the specificity of any altered perceptual patterns in autism.

\subsection{Facelobject processing in autism and the relationship to configural processing}

In the course of this paper, three series of studies were undertaken. The first set revealed that 14 autistic adults were sig- nificantly slowed at making same/different discriminations between novel faces, relative to their matched control counterparts, and this was so to a greater extent as the perceptual discrimination was more fine-grained. The second set of studies explored the configural processing abilities of the same autistic subjects. Relative to the controls, the autistic individuals show a local bias or local superiority such that identifying local elements was faster than identifying global letters or shapes and identifying a compound letter at the global level was slowed by inconsistent local information.

A more detailed investigation, manipulating the number of elements in a display and the time course by which the local versus global information is available, revealed a pattern of behavior that differs from the well-established profile of neurotypical individuals. In particular, the autistic individuals did not show the signature effects of normal grouping behavior in displays with many items: early priming for test pairs that are configurally similar to the prime (CS) over items that are similar to the prime in the local elements (ES) reflecting the global advantage with many elements (Kimchi, 1998) at brief exposure durations (Navon, 1977; Paquet \& Merikle, 1984). Instead, the autistic individuals are primed by the local elements and show a clear advantage for the elements over the whole shape. Taken together, the large local-to-global interference in the global/local experiment, and the absence of facilitation from the global configuration in the primed matching experiment (in the many-element condition) at any of the possible prime durations (not just an early configural organization) suggest that the autistic subjects may be able to derive a global configuration, in a time-consuming fashion, only under favorable circumstances (facilitating local information) and that, in general, they show a bias towards local elements.

We also confirmed that the poorer perceptual performance in autism is not attributable to a fundamental difference in spatial frequency thresholds. Of particular interest is that the difficulty in configural processing, indexed by the failure to group rapidly and efficiently many relatively small elements into a global shape, was positively correlated with the slowing in face processing. Although our focus has been on visual processing, we note the visual perceptual difficulty we have documented here may be part of a more general perceptual pattern; the apparent difficulty in detecting configurations in the auditory modality (Foxton et al., 2003) also suggests a bias towards local processing and may reflect a pattern in autism that is independent of the sensory modality of the input.

The third set of studies showed that the perceptual differences in autism extended beyond faces to non-face objects too, with the autistic individuals being significantly slowed, relative to the controls, on same/different discrimination tasks. This slowing was not as great as was true for faces and it did not hold perfectly consistently across all individuals and all conditions. A positive correlation was also noted between the local bias and the performance on the Greeble processing task but not on the non-face object 
task. Taken together, these findings indicate important differences in visuoperceptual processing in autism compared with the control subjects in both face/non-object and in configural processing and further suggest that there may be a positive relationship between these patterns of performance.

\subsection{Preference for local information in autism}

The obvious question is what underlying mechanism gives rise to these differences in perceptual performance. We have shown that there are no differences in spatial frequency thresholds in autism and in the control group, suggesting that the differences are probably not arising at the very early stages of the visual system. Consistent with this, a recent functional magnetic resonance imaging study found no differences between the sensory visual areas of people with autism and normal controls (Hadjikani et al., 2004) - the ratio of central to peripheral visual field representation was normal and the maps of retinotopic organization did not differ from those of controls in any respect. This result would suggest that the visual abnormalities arise further upstream and that the process involved in the perceptual organization and grouping of the local elements may be the source of the difference. However, we should note that several studies have argued for a low-level visual deficit in autism in the domain of motion perception; given random dot stereograms with the requirement to detect coherent motion, individuals with autism have higher thresholds for detection than their peers (Milne et al., 2002), are deficient in motion direction discrimination (Bertone, Mottron, Jelenic, \& Faubert, 2003) and experience difficulty with rapidly moving stimuli (Gepner \& Mestre, 2002). Although these biases have been attributed to an alteration in the magnocellular pathways in autism (Milne et al., 2002), they may possibly be reinterpreted as a higher-order difficulty in integrating elements, reflecting the same local bias as we have documented here. Determining motion coherence or patterns of biological motion requires the observer to track multiple parts of the display and the relationship of the local elements with each other. Additionally, items must be integrated across time and the difficulty may arise in this integration, just as we have demonstrated a deficit in integrating local elements across space. Thus, the motion deficit might potentially also arise from the failure to derive a global configuration from stimuli $^{3}$ containing multiple local elements and not from a change in early visual processing per se.

We have suggested that the apparent slowing in object processing, and to a greater extent in face processing may be explained by the local bias exhibited by the autistic individuals. The relationship between the ability to configure local

\footnotetext{
3 On the surface, one possible exception to this is that Blake and colleagues (Blake, Turner, Smoski, Pozdol, \& Stone, 2003) report increased biological motion thresholds in autistic children with preserved ability to group small line elements into a single global figure. This form of grouping, by collinearity, is thought to be done by early visual processes (Kovács, Kozma, Feher, \& Benedek, 1999) and can be distinguished from the kind of higher-level grouping into identifiable shapes to which we are referring.
}

elements and face processing is well-established in other populations. There are several studies showing that individuals with prosopagnosia perform poorly on tasks that require the integration of perceptual information (Barton et al., 2002) and integrative agnosic patients who are impaired at grouping information to form a global shape may show concurrent problems in object and face recognition (Behrmann \& Kimchi, 2003; Ricci, Vaishnavi, \& Chatterjee, 1999). Moreover, individuals who are congenitally prosopagnosic (CP), with no identifiable neural substrate that gives rise to this behavioral alteration, are impaired on the global/local task and show a profile similar to that of the autistic individuals on the very same few/many experiment conducted here (Behrmann et al., 2005). We note that these $\mathrm{CP}$ individuals also differ from their controls on the non-face objects, as is the case for the autistic individuals tested here. The CP individuals, however, do not share the neuropsychological and social deficits present in most autistic individuals. These sources of evidence from other populations bolster the claim that there is a relationship between face and configural processing. But, of course, correlation is not causation and causality still remains to be determined.

In sum, the view that we have taken here is that the slowing in face processing in autism might arise from a more fundamental visual (and possibly even sensory independent) bias towards the local elements and perhaps simultaneous or resultant difficulty in integrating local components of a stimulus into a whole. Moreover, this fundamental perceptual form of processing is not restricted to faces but may impact visual processing of other non-face objects too when the demands for discrimination and recognition are high, as is true in the case of faces. Because faces are typically identified at an exemplar-specific level, they are the most susceptible to the impairment in integrating local elements, but common objects and novel Greebles are also affected, albeit not to the same degree as faces.

In contrast with this view of weak perceptual coherence or difficulty comprehending the spatial relationship between the elements perhaps by virtue of a local bias, other studies have argued that the face processing difficulties in autism arise as a consequence of a social, rather than perceptual, deficit. For example, it is known that individuals with autism have a limited capacity for social adaptation (Klin et al., 2002) and orient more to objects than to faces (Dawson et al., 2004). But it is possible that the social deficit and the perceptual disorder work in tandem: the lack of experience and the inadequate attention to faces may limit the acquisition of the normal configural perceptual skill. Consistent with the claim that expertise comes to fine-tune or optimize the fusiform gyrus (FG), the putative 'face area', as a function of experience with a class of stimuli (Gauthier et al., submitted for publicationGauthier, Tarr, Anderson, Skudlarski, \& Gore, 1999; Le Grand, Mondloch, Maurer, \& Brent, 2003), the fusiform region of autistic individuals does not apparently come to respond preferentially to faces and this hypoactivation may be a direct reflection of the social disability 
(Schultz, 2005; Schultz et al., 2003). Some, although not all, recent functional magnetic resonance imaging studies have shown reduced activation of the FG of autistic individuals when viewing faces; instead strong activation is noted in the inferior temporal gyrus region, the region activated during object discrimination in controls (Grelotti et al., 2005; Hubl et al., 2003; Pierce et al., 2004, 2001; Schultz et al., 2000).

We have, however, suggested an alternative interpretation and that it is that face processing is slowed not solely because faces are social but because they represent a particularly complex visual stimulus that depends specifically on configural processing. This view is also supported by a recent study that shows that face recognition is not correlated with ratings of social impairment nor does it line up with a particular diagnosis of social developmental disorder. Instead, as we do, the authors argue that any difficulties in face processing in individuals with social deficits may well be causally related to an underlying perceptual alteration, suggestive of occipitotemporal dysfunction (Barton et al., 2004). Whether the perceptual alteration is primarily responsible for the local bias and/or difficulty to derive configuration or whether it comes from lack of experience with faces remains to be determined. Of course, the social disability and the perceptual performance are not mutually exclusive and each may ultimately contribute both to the difficulties in processing faces and the difficulties in configural processing. The challenge is to understand their relative and joint contributions in greater detail.

\subsection{Caveats and considerations}

We have argued for an altered perceptual profile in autism based on differences in reaction time between neurotypical and autistic subjects in face and object processing as well as in configural processing. We have suggested that these reaction time differences provide an important index of the nature of visual processing in autism. Before concluding, however, we need to consider some limitations of our approach and, at the same time, caution the reader to possible confounds that might be at play. The experiments were all conducted in a 'data-unlimited fashion', i.e., the stimuli were present for an unlimited duration and our dependent measure was the speed with which the various perceptual decisions were made rather than the accuracy, as would be more telling in cases of displays with brief presentation of visual images. Our approach, while advantageous in approximating naturalistic conditions where stimuli remain present for extended periods of time, is potentially problematic, too. One complication is that just because autistic subjects are slower than the controls does not obviously tell us whether the pattern of data in autism is qualitatively different rather than quantitatively different. Indeed, autistic individuals are notoriously slow in their motor control and response time (Bauman, 1992). Emphasizing the interaction (for example, greater slowing in condition B than A for autistics than for controls), while somewhat helpful, does not eliminate this quandary, and in- stead, introduces questions about confounds that emerge from artifacts of scaling (a problem that has plagued literature such as that in the domain of schizophrenia). In some cases and in some of our paradigms, we have been able to show qualitative rather than simply quantitative differences. For example, in the global/local task, the control subjects show global superiority whereas the autistic subjects exhibit local superiority. A second example is in the few/many task in which autistic individuals (under the range of temporal intervals used here) never exhibit priming (equal or better performance on CS than ES) when primes share configural representations with the probe whereas this is evident in normal individuals both early and late with many element displays. However, the pattern of data in the face, object and Greeble tasks all indicate slowing in autism compared with control and understanding the basis of this slowing will require further investigation. Converging evidence from studies using brief exposure and accuracy may help in this regard.

An additional issue to confront is the apparent discrepancies between various measures in the paradigms we have reported. So, for example, while statistically significant group differences may be observed (as in object and Greeble experiment), more detailed analyses do not always bear out the predictions. It is also the case that not every individual autistic subject shows the full profile of perceptual differences (see Table 2). These issues suggest that one might want to exercise some caution in interpreting the data and, indeed, we recommend that additional data be collected to verify the strength of the patterns we have observed.

Even if we set aside these statistical/technical issues, there still remains the question of the mechanism underlying these differences. As stated above, the differences could arise in the perceptual system of autistic individuals or even in the motor response system. The differences might also reflect a preferential strategy or cognitive style in which autistic individuals spend more time inspecting details in a situation when there is no requirement for speed in a task. Why these individuals adopt this style or strategy still remains an open issue but, importantly, if this were the case, the slowed performance might arise neither in the perceptual nor in the motor systems per se and an alternative approach entirely might need to be sought.

Another facet of the data that requires cautious interpretation is that the match between the control group and autistic groups was not perfect. We had not been able to match the groups on IQ but matched on age and gender and education level (as a rough but obviously not adequate proxy for intellectual function). Although we analyzed the data from the highest functioning autistic individuals in some sub-analyses and showed that the pattern of data remained unchanged compared with the entire group analysis, the need for a comparable intellectual group is pressing to ensure definitively that the patterns we see are specific to autism and not attributable to differential intellectual competence between the autistic and control group.

A final, more theoretical question that remains to be addressed is exactly what constitutes 'configural' processing 
and whether the configural processing required for faces (and for other objects) is the same as that required for global/local and few/many element processing. The definition of configural processing is highly controversial and is the focus of many investigations (Gauthier \& Tarr, 2002; Leder \& Bruce, 2000; Maurer et al., 2002; Moscovitch, Winocur, \& Behrmann, 1997). In the domain of face perception, one sense of "configural' refers to the perception of relations among the features of a stimulus, with a distinction between two types of configural processing (Carey \& Diamond, 1994; Rhodes, 1988): (i) first-order relations among elements, for example, processing the presence of two eyes above a nose and (ii) secondorder relations-local elements are processed in a relational manner (e.g., nose-mouth distance). A second sense of the term 'configural' refers to holistic processing - the features or local elements are glued together into a gestalt (Tanaka \& Sengco, 1997). Although the distinctions are reasonable, many outstanding issues remain such as the extent of the superadditivity of the features in the holistic case, the ability to access the local elements in the holistic case and the relationship between these different forms. Additionally, these various configural processes are generally considered in relation to faces (but see (Gauthier \& Tarr, 2002)) and so applying this taxonomy to the global/local task or to the few/many task is less clear. Understanding what constitutes configural processing, how the elements are represented in relation to each other and whether the same form of configural processing applies across all visual stimuli is critical for future research. Moreover, detailed understanding of the configural processing is autism remains elusive but its specification is a necessary precursor to developing targeted intervention procedures.

\section{Acknowledgements}

This research was funded by a grant from the NICHD/NIDCD PO1/U19 to Marlene Behrmann (PI: Nancy Minshew), which is part of the NICHD/NIDCD Collaborative Programs for Excellence in Autism and by grants from the National Alliance of Autism Research and the Cure Autism Now Foundation to Kate Humphreys.

\section{References}

Bacon, W. F., \& Egeth, H. E. (1991). Local processes in pre-attentive feature detection. Journal of Experimental Psychology: Human Perception and Performance, 17, 77-90.

Badcock, C. J., Whitworth, F. A., Badcock, D. R., \& Lovegrove, W. J. (1990). Low-frequency filtering and processing of local-global stimuli. Perception, 19, 617-629.

Barton, J. J. S., Cherkasova, M. V., Hefter, R., Cox, T. A., O’Connor, M., \& Manoach, D. S. (2004). Are patients with social developmental disorders prosopagnosic? Perceptual heterogeneity in the Asperger and social-emotions processing disorders. Brain, 127(8), 1706-1716.

Barton, J. J. S., Press, D. Z., Keenan, J. P., \& O'Connor, M. (2002). Lesions of the fusiform face area impair perception of facial configuration in prosopagnosia. Neurology, 58, 71-78.
Bauman, M. L. (1992). Motor dysfunction in autism. In A. B. Joseph \& R. R. Young (Eds.), Movement disorders in neurology and psychiatry (pp. 658-661). Boston, MA: Blackwell.

Behrmann, M., \& Kimchi, R. (2003). What does visual agnosia tell us about perceptual organization and its relationship to object perception? Journal of Experimental Psychology: Human Perception and Performance, 29(1), 19-42.

Behrmann, M., Avidan, G., Marotta, J. J., \& Kimchi, R. (2005). Detailed exploration of face-related processing in congenital prosopagnosia: 1. Behavioral findings. Journal of Cognitive Neuroscience, 17(7), 1-19.

Beller, H. K. (1971). Priming: Effects of advance information on matching. Journal of Experimental Psychology, 87, 176-182.

Bertone, A., Mottron, L., Jelenic, P., \& Faubert, J. (2003). Motion perception in autism: A complex issue. Journal of Cognitive Neuroscience, 15(2), 218-225.

Blair, R. J. R., Frith, U., Smith, N., Abell, F., \& Cipolotti, L. (2001). Fractionation of visual memory: Agency detection and its impairment in autism. Neuropsychologia, 40, 108-118.

Blake, R., Turner, L. M., Smoski, M. J., Pozdol, S. L., \& Stone, W. L. (2003). Visual recognition of biological motion is impaired in children with autism. Psychological Science, 14(2), 150-157.

Booth, R., Charlton, R., Hughes, C., \& Happé, F. (2003). Disentangling weak coherence and executive dysfunction: Planning drawing in autism and attention-deficit/hyperactivity disorder. Philosophical Transactions of Royal Society of London. Series B: Biological Sciences, 358(1430), 387-392.

Boucher, J., \& Lewis, V. (1992). Unfamiliar face recognition in relatively able autistic children. Journal of Child Psychology and Psychiatry, 33(5), 843-859.

Brosnan, M. J., Scott, F. J., Fox, S., \& Pye, J. (2004). Gestalt processing in autism: Failure to process perceptual relationships and the implications for contextual understanding. Journal of Child Psychology and Psychiatry, 45(3), 459-469.

Carey, S., \& Diamond, R. (1994). Are faces perceived as configurations more by adults than by children? Visual Cognition, 1(2-3), 253-274.

Caron, M. J., Mottron, L., Rainville, C., \& Chouinard, S. (2004). Do high functioning persons with autism present superior spatial abilities? Neuropsychologia, 42(4), 467-481.

Cohen, J. D., MacWhinney, B., Flatt, M., \& Provost, J. (1993). Psyscope: A new graphic interactive environment for designing psychology experiments. Behavioral Research Methods, Instruments and Computers, 25(2), 257-271.

Crawford, J. R., \& Garthwaite, P. H. (2004). Statistical methods for single-case studies in neuropsychology: Comparing the slope of a patient's regression line with those of a control sample. Cortex, 40, 533548.

Critchley, H. D., Daly, E. M., Bullmore, E. T., Williams, S. C. R., Amelsvoort, T. V., Robertson, D. M., et al. (2000). The functional neuroanatomy of social behaviour: Changes in cerebral blood flow when people with autistic disorder process facial expressions. Brain, 123, 2203-2212.

Cumming, G., \& Finch, S. (2005). Inference by eye: Confidence intervals and how to read pictures of data. American Psychologist, 60(2), 170-180.

Dalton, K. M., Nacewicz, B. M., Johnstone, T., Schaefer, H. S., Gernsbacher, M. A., Goldsmith, H. H., et al. (2005). Gaze fixation and the neural circuitry of face processing in autism. Nature Neuroscience, $8(4), 519-526$.

Davies, S., Bishop, D., Manstead, A. S. R., \& Tantum, D. (1994). Face perception in children with autism and Asperger's syndrome. Journal of Child Psychology and Psychiatry, 35(6), 1033-1057.

Dawson, G., Carver, L., Meltzoff, A. N., Panagiotides, H., McPartland, J., \& Webb, S. J. (2002). Neural correlates of face and object recognition in young children with autism spectrum disorder, developmental delay, and typical development. Child Development, 73(3), 700-717.

Dawson, G., Toth, K., Abbott, R., Osterling, J., Munson, J., Estes, A., et al. (2004). Early social attention impairments in autism: Social ori- 
enting, joint attention, and attention to distress. Developmental Psychology, 40(2), 271-283.

Delvenne, J.-F., Seron, X., Coyette, F., \& Rossion, B. (2004). Evidence for perceptual deficits in associative visual (prosop)agnosia: A single case study. Neuropsychologia, 42(5), 597-612.

Ellis, H. D., Ellis, D. M., Fraser, W., \& Deb, S. (1994). A preliminary study of right hemisphere cognitive deficits and impaired social judgments among young people with Asperger syndrome. European Child and Adolescent Psychiatry, 3, 255-266.

Farah, M. J., Tanaka, J. W., \& Drain, H. M. (1996). What causes the face inversion effect? Journal of Experimental Psychology: Human Perception and Performance, 21(3), 1-7.

Farah, M. J., Wilson, K. D., Drain, H. M., \& Tanaka, J. R. (1995). The inverted face inversion effect in prosopagnosia: Evidence for mandatory, face-specific perceptual mechanisms. Vision Research, 35, 2089-2093.

Foxton, J. M., Stewart, M. E., Barnard, L., Rodgers, J., Young, A. H., O'Brien, G., et al. (2003). Absence of auditory 'global interference' in autism. Brain, 126(Pt 12), 2703-2709.

Frith, U. (2003). Autism: Explaining the enigma. UK: Blackwell Publishers.

Frith, U., \& Happé, F. G. E. (1994). Autism: Beyond 'theory of mind'. Cognition, 50, 115-132.

Gauthier, I., \& Tarr, M. J. (1997). Becoming a "greeble" expert: Exploring mechanisms for face recognition. Vision Research, 37(12), 1673-1682.

Gauthier, I., \& Tarr, M. J. (2002). Unraveling mechanisms for expert object recognition: Bridging brain activity and behavior. Journal of Experimental Psychology: Human Perception and Performance, 28(2), $431-446$.

Gauthier, I., Tarr, M. J., Anderson, A., Skudlarski, P., \& Gore, J. (1999). Activation of the middle fusiform 'face area' increases with expertise for recognizing novel objects. Nature Neuroscience, 2, 568-573.

Gauthier, I., Behrmann, M., \& Tarr, M. J. (1999). Can face recognition really be dissociated from object recognition? Journal of Cognitive Neuroscience, 11(4), 349-370.

Gepner, B., \& Mestre, D. (2002). Rapid visual-motion integration deficit in autism. Trends in Cognitive Sciences, 6(11), 455.

Gerlach, C., Marstrand, L., Habekost, T., \& Gade, A. A case of impaired shape integration: Implications for models of visual object recognition. Visual Cognition, in press.

Ginsburg, A. P. (1986). Spatial filtering and visual form information. In K. R. Boff, L. Kaufman, \& J. P. Thomas (Eds.), Handbook of Human Perception and Performance (pp. 1-41). New York: Wiley.

Grelotti, D. J., Klin, A. J., Gauthier, I., Skudlarski, P., Cohen, D. J., Gore, J. C., et al. (2005). fMRI activation of the fusiform gyrus and amygdala to cartoon characters but not to faces in a boy with autism. Neuropsychologia, 43(3), 373-385.

Grice, S. J., Spratling, M. W., Karmiloff-Smith, A., Halit, H., Csibra, G., de Haan, M., et al. (2001). Disordered visual processing and oscillatory brain activity in autism and Williams syndrome. Neuroreport, 28(12), 2697-2700.

Hadjikani, N., Chabris, C. F., Joseph, R. M., Clark, J., McGrath, L., Aharon, I., et al. (2004). Early visual cortex organization in autism-An fMRI study. Neuroreport, 15(2), 267-270.

Happé, F. G. E. (1996). Studying weak central coherence at low levels: Children with autism do not succumb to visual illusions. Journal of Child Psychology and Psychiatry, 37(7), 873-877.

Happé, F. G. E. (1999). Autism: Cognitive deficit or cognitive style? Trends in Cognitive Sciences, 3(6), 216-222.

Hauck, M., Fein, D., Maltby, N., Waterhouse, L., \& Feinstein, C. (1998). Memory for faces in children with autism. Child Neuropsychology, 4, 187-198.

Hobson, R. P. (1986). The autistic child's appraisal of expressions of emotion: A further study. Journal of Child Psychology and Psychiatry, 27(5), 671-680.

Hobson, R. P. (1987). The autistic child's recognition of age- and sexrelated characteristics of people. Journal of Autism and Developmental Disorder, 17(1), 63-79.
Hobson, R. P., Ouston, J., \& Lee, A. (1988). What's in a face? The case of autism. British Journal of Psychology, 79, 441-453.

Hubl, D., Bolte, S., Feineis-Matthews, S., Lanfermann, H., Federspiel, A., Strik, W., et al. (2003). Functional imbalance of visual pathways indicates alternative face processing strategies in autism. Neurology, 61(9), 1232-1237.

Hughes, H. C., Fendrich, R., \& Reuter-Lorenz, P. (1990). Global versus local processing in the absence of low spatial frequencies. Journal of Cognitive Neuroscience, 2, 272-282.

Jolliffe, T., \& Baron-Cohen, S. (1997). Are people with autism and asperger syndrome faster than normal on the embedded figures test? Journal of Child Psychology and Psychiatry, 38(5), 527-534.

Joseph, R. M., \& Tanaka, J. (2003). Holistic and part-based face recognition in children with autism. Journal of Child Psychology and Psychiatry, 44(4), 529-542.

Kanner, L. (1943). Autistic disturbance of affective contact. Nervous Child, 2, 217-240.

Kaufman, A. S., \& Kaufman, N. L. (1985). Kaufman test of educational achievement. Circle Pines, MN: American Guidance Service.

Kimchi, R. (1998). Uniform connectedness and grouping in the perceptual organization of hierarchical patterns. Journal of Experimental Psychology: Human Perception and Performance, 24(2), 1105-1118.

Kimchi, R. (2000). The perceptual organization of visual objects: A microgenetic analysis. Vision Research, 40, 1333-1347.

Klin, A., Jones, W., Schultz, R., Volkmar, F., \& Cohen, D. (2002). Defining and quantifying the social phenotype in autism. American Journal of Psychiatry, 159, 895-908.

Klin, A., Sparrow, S. S., de Bildt, A., Cicchetti, D. V., Cohen, D. J., \& Volkmar, F. R. (1999). A normed study of face recognition in autism and related disorders. Journal of Autism and Development Disorders, 29(6), 499-508.

Kovács, I., Kozma, P., Feher, A., \& Benedek, G. (1999). Late maturation of visual spatial integration in humans. Proceedings of the National Academy of Sciences, 96(21), 12204-12209.

Lamb, M., \& Robertson, L. (1988). The processing of hierarchical stimuli: Effects of retinal locus, location uncertainty, and stimulus identity. Perception and Psychophysics, 44, 172-181.

Lamb, M., \& Yund, E. W. (1993). The role of spatial frequency in the processing of hierarchically organized structure. Perception and Psychophysics, 54, 773-784.

Langdell, T. (1977). Recognition of faces: An approach to the study of autism. Journal of Child Psychology and Psychiatry, 19, 255268.

Le Couteur, A., Rutter, M., Lord, C., Rios, P., Robertson, S., Holdgrafer, M., et al. (1989). Autism diagnostic interview: A standardized investigator-based instrument. Journal of Autism and Developmental Disorders, 19, 363-387.

Le Grand, R., Mondloch, C. J., Maurer, D., \& Brent, H. P. (2003). Expert face processing requires visual input to the right hemisphere during infancy. Nature Neuroscience, 6(10), 1108-1112.

Le Grand, R., Mondloch, C. J., Maurer, D., \& Brent, H. P. (2004). Impairment in holistic face processing following early visual deprivation. Psychological Sciences, 15(11), 762-768.

Leder, H., \& Bruce, V. (2000). When inverted faces are recognized: The role of configural information in face recognition. Quarterly Journal of Experimental Psychology A, 53(2), 513-536.

Lopez, B., Donnelly, N., \& Hadwin, J. A. (2004). Face processing in high-functioning adolescents with autism: Evidence for weak central coherence. Visual Cognition, 11(6), 673-688.

Lord, C., Rutter, M., Goode, S., Heemsbergen, J., Jordan, H., Mawhood, L., et al. (1989). Autism diagnostic observation schedule: A standardized observation of communicative and social behavior. Journal of Autism and Developmental Disorders, 19, 185-212.

Lord, C., Rutter, M., \& Le Couteur, A. (1994). Autism diagnostic interview-revised: A revised version of a diagnostic interview for caregivers of individuals with possible pervasive developmental disorders. Journal of Autism and Developmental Disorders, 24, 659-685. 
Marotta, J. J., McKeeff, T. J., \& Behrmann, M. (2002). The effects of inversion and rotation on face processing in prosopagnosia. Cognitive Neuropsychology, 19(1), 31-47.

Martin, M. (1979). Local and global processing: The role of sparsity. Memory \& Cognition, 7, 476-484.

Maurer, D., Le Grand, R., \& Mondloch, C. J. (2002). The many faces of configural processing. Trends in Cognitive Sciences, 6(6), 255-260.

McPartland, J., Dawson, G., Webb, S. J., Panagiotides, H., \& Carver, L. J. (2004). Event-related brain potentials reveal anomalies in temporal processing of faces in autism spectrum disorder. Journal of Child Psychology and Psychiatry, 45(7), 1235-1245.

Miller, M. B., Chapman, J. P., Chapman, L. J., \& Collins, J. (1995). Task difficulty and cognitive deficits in schizophrenia. Journal of Abnormal Psychology, 104(2), 251-258.

Milne, E., Swettenham, J., Hansen, P., Campbell, R., Jeffries, H., \& Plaisted, K. (2002). High motion coherence thresholds in children with autism. Journal of Child Psychology and Psychiatry, 43(2), 255-263.

Minshew, N., Goldstein, G., \& Siegel, D. (1997). Neuropsychological functioning in autism: Profile of a complex information processing disorder. Journal of the International Neuropsychological Society, 3, 303-316.

Moscovitch, M., \& Moscovitch, D. A. (2000). Super face-inversion effects for isolated internal or external features, and for fractured faces. Cognitive Neuropsychology, 17(1-3), 201-220.

Moscovitch, M., Winocur, G., \& Behrmann, M. (1997). What is special about face recognition? Nineteen experiments on a person with visual object agnosia and dyslexia but normal face recognition. Journal of Cognitive Neuroscience, 9(5), 555-604.

Mottron, L., \& Belleville, S. (1993). A study of perceptual analysis in high-level autistic subject with exceptional graphic abilities. Brain and Cognition, 23, 279-309.

Mottron, L., Burack, J. A., Iarocci, G., Belleville, S., \& Enns, J. T. (2003). Locally oriented perception with intact global processing among adolescents with high-functioning autism: Evidence from multiple paradigms. Journal of Child Psychology and Psychiatry, 44(6), 904-913.

Mottron, L., Burack, J., Stauder, J., \& Robaey, P. (1999). Perceptual processing among high-functioning persons with autism. Journal of Child Psychology and Psychiatry and Allied Disciplines, 40(2), 203-211.

Navon, D. (1977). Forest before trees: The precedence of global features in visual perception. Cognitive Psychology, 9, 353-383.

Navon, D. (2003). What does a compound letter tell the psychologist's mind? Acta Psychologica (Amsterdam), 114(3), 273-309.

Njiokiktjien, C., Verschoor, A., de Sonneville, L., Huyser, C., Op het Veld, V., \& Toorenaar, N. (2001). Disordered recognition of facial identity and emotions in three Asperger type autists. European Child and Adolescent Psychiatry, 10(1), 79-90.

Ozonoff, S., Strayer, D. L., McMahon, W. M., \& Filloux, F. (1994). Executive function abilities in autism and Tourette syndrome: An information processing approach. Journal of Child Psychology and Psychiatry, 35(6), 1015-1032.

Paquet, L., \& Merikle, P. M. (1984). Global precedence: The effect of exposure duration. Canadian Journal of Psychology, 38, 45-53.

Pierce, K., Haist, F., Sedaghat, F., \& Courchesne, E. (2004). The brain response to personally familiar faces in autism: Findings of fusiform activity and beyond. Brain, 127, 2703-2716.

Pierce, K., Muller, R. A., Ambroses, J., Allen, G., \& Courchesne, E. (2001). Face processing occurs outside the fusiform 'face area' in autism: Evidence from fMRI. Brain, 124, 2059-2073.

Plaisted, K. (2000). Aspects of autism that theory of mind cannot explain. In S. Baron-Cohen, H. Tager-Flusberg, \& D. Cohen (Eds.), Understanding other minds: Perspectives from developmental cognitive neurosciences (pp. 224-250). New York: Oxford Press.

Plaisted, K., O'Riordan, M., \& Baron-Cohen, S. (1998). Enhanced discrimination of novel, highly similar stimuli by adults with autism during a perceptual learning task. Journal of Child Psychology and Psychiatry, 39(5), 765-775.
Plaisted, K., Saksida, L., Alcantara, J., \& Weisblatt, E. (2003). Towards an understanding of the mechanisms of weak central coherence effects: Experiments in visual configural learning and auditory perception. Philosophical Transactions of Royal Society of London. Series B: Biological Sciences, 358(1430), 375-386.

Plaisted, K., Swettenham, J., \& Rees, L. (1999). Children with autism show local precedence in a divided attention task and global precedence in a selective attention task. Journal of Child Psychology and Psychiatry and Allied Disciplines, 40(5), 733-742.

Pomerantz, J. R. (1983). Global and local precedence: Selective attention in form and motion perception. Journal of Experimental Psychology: General, 112(4), 516-540.

Rhodes, G. (1988). Looking at faces: First-order and second-order features as determinates of facial appearance. Perception, 17, 43-63.

Ricci, R., Vaishnavi, S., \& Chatterjee, A. (1999). A deficit of intermediate vision: Experimental observations and theoretical implications. Neurocase, 5, 1-12.

Rinehart, N., Bradshaw, J., Moss, S., Brereton, A., \& Tonge, B. (2000). Atypical interference of local detail on global processing in highfunctioning autism and Asperger's disorders. Journal of Child Psychology and Psychiatry and Allied Disciplines, 41(6), 769-788.

Ropar, D., \& Mitchell, P. (1999). Are individuals with autism and asperger's syndrome susceptible to visual illusions? Journal of Child Psychology and Psychiatry, 40(8), 1283-1293.

Rouse, H., Donnelly, N., Hadwin, J. A., \& Brown, T. (2004). Do children with autism perceive second-order relational features? The case of the Thatcher illusion. Journal of Child Psychology and Psychiatry, 45(7), 1246-1257.

Schultz, R. T. (2005). Developmental deficits in social perception in autism the role of the amygdala and fusiform face area. International Journal of Developmental Neuroscience, 23, 125-141.

Schultz, R. T., Gauthier, I., Klin, A., Fulbright, R. K., Anderson, A. W., Volkmar, F., et al. (2000). Abnormal ventral temporal cortical activity during face discrimination among individuals with autism and Asperger syndrome. Archives of General Psychiatry, 57, 331-340.

Schultz, R. T., Grelotti, D. J., Klin, A., Kleinman, J., Van der Gaag, C., Marois, R., et al. (2003). The role of the fusiform face area in social cognition: Implications for the pathobiology of autism. Philosophical Transactions of Royal Society of London. Series B: Biological Sciences, 358(1430), 415-427.

Seitz, K. (2002). Parts and wholes in person recognition: Developmental trends. Journal of Experimental Child Psychology, 82(4), 367-381.

Sekuler, A. B., \& Palmer, S. E. (1992). Perception of partly occluded objects: A microgenetic analysis. Journal of Experimental Psychology: General, 121(1), 95-111.

Shah, A., \& Frith, U. (1983). An islet of ability in autism: A research note. Journal of Child Psychology and Psychiatry, 24, 613-620.

Shulman, G. L., \& Wilson, J. (1987). Spatial frequency and selective attention to local and global information. Neuropsychologia, 18, 89-101.

Shulman, G. L., Sullivan, M. A., Gish, K., \& Sakoda, W. J. (1986). The role of spatial-frequency channels in the perception of local and global structure. Perception, 15, 259-273.

Society for Autistic Children. (1978). National society for autistic children definition of the syndrome of autism. Journal of Autism and Development Disorder, 8, 162-167.

Tanaka, J. W., \& Farah, M. J. (1993). Parts and wholes in face recognition. Quarterly Journal of Experimental Psychology, 46A, 225-245.

Tanaka, J. W., \& Sengco, J. A. (1997). Features and their configuration in face recognition. Memory and Cognition, 25, 583-592.

Tantam, D., Monaghan, L., Nicholson, H., \& Stirling, J. (1989). Autistic children's ability to interpret faces: A research note. Journal of Child Psychology and Psychiatry, 30(4), 623-630.

Tarr, M. J., \& Cheng, Y. D. (2003). Learning to see faces and objects. Trends in Cognitive Science, 7(1), 23-30.

Teunisse, J., \& De Gelder, B. (2003). Face processing in adolesents with autistic disorder: The inversion and composite effects. Brain and $\mathrm{Cog}$ nition, 32, 285-294. 
Volkmar, F. R., Lord, C., Bailey, A., Schultz, R. T., \& Klin, A. (2004). Autism and pervasive developmental disorders. Journal of Child Psychology and Psychiatry, 45(1), 135-170.

Wechsler, D. (1997). Wechsler intelligence scale (third ed.). San Antonio, TX: The Psychological Corporation.
Yin, R. K. (1969). Looking at upside-down faces. Journal of Experimental Psychology, 81, 141-145.

Yovel, G., Paller, K. A., \& Levy, J. (2005). A whole face is more than the sum of its halves: Interactive processing in face perception. Visual Cognition, 12(2), 337-352. 